\title{
Combinatorial mapping-torus, branched surfaces and free group automorphisms
}

\author{
FRANÇOIS GAUTERO
}

\begin{abstract}
We give a characterization of the geometric automorphisms in a certain class of (not necessarily irreducible) free group automorphisms. When the automorphism is geometric, then it is induced by a pseudo-Anosov homeomorphism without interior singularities. An outer free group automorphism is given by a 1-cocycle of a 2-complex (a standard dynamical branched surface, see [7] and [9]) the fundamental group of which is the mapping-torus group of the automorphism. A combinatorial construction elucidates the link between this new representation (first introduced in [16]) and the classical representation of a free group automorphism by a graph-map [2].
\end{abstract}

Mathematics Subject Classification (2000): 20E05, 57M20, 37Bxx, 37E25.

\section{Introduction}

Let $S$ be a compact surface, with fundamental group $\pi$. Any homeomorphism $h$ of $S$ induces an outer automorphism $h_{\#}$ of $\pi$, which only depends on the isotopy-class of $h$. A classical question is to know to what extent we can go back from an outer automorphism of a surface group $\pi$, i.e. a group isomorphic to the fundamental group of a compact surface, to an isotopy-class of surface homeomorphisms. More precisely, let $\pi$ be a surface group and let $\alpha \in \operatorname{Out}(\pi)=\operatorname{Aut}(\pi) / \operatorname{Inn}(\pi)$; does there exist a compact surface $S$ with fundamental group $\pi$ and a homeomorphism $h$ of $S$ such that $h_{\#}=\alpha$ ? If so, then we say that $\alpha$ (or any automorphism in the class) is geometric.

If $\pi$ is isomorphic to the fundamental group of a compact surface with empty boundary, then this surface is unique up to homeomorphism and an old result of Nielsen tells us that any $\alpha \in \operatorname{Out}(\pi)$ is geometric. Assume now that $\pi$ is isomorphic to the fundamental group of a compact surface with boundary, i.e. $\pi$ is a rank- $n$ free group, denoted by $\mathbb{F}_{n}$. Up to homeomorphism, there are a finite number of distinct surfaces with fundamental group $\mathbb{F}_{n}$. Is any element of $\operatorname{Out}\left(\mathbb{F}_{n}\right)$ geometric? Nielsen again (see for instance $[10,11]$ ) provides an answer: "the automorphism $\alpha$ is geometric if and only if there exists a free basis $\mathcal{B}$ of $\mathbb{F}_{n}$ such that $\alpha$ preserves, up to reduction and change of orientation, a set of reduced words in $\mathcal{B}$ whose union contains exactly twice each element of $\mathcal{B}$ ". A reduced word in an

Received September 18, 2006; accepted in revised form June 25, 2007. 
alphabet $\left\{e_{i}^{ \pm 1}\right\}_{i=1, \cdots, n}$ is a word with no cancellation $e_{i} e_{i}^{-1}$ or $e_{i}^{-1} e_{i}$. For $n=2$, this condition is always satisfied (any $\alpha \in \operatorname{Out}\left(\mathbb{F}_{2}\right)$ is induced by a homeomorphism of the torus deprived of an open disc) but for $n \geq 3$, geometric automorphisms of $\mathbb{F}_{n}$ are "rare" [18].

The above answer is however not completely satisfactory. Because of the words "there exists" emphasized above, given $\alpha \in \operatorname{Out}\left(\mathbb{F}_{n}\right)$, it does not provide a way to detect whether $\alpha$ is geometric. The problem of finding other characterizations of these geometric free group automorphisms was already addressed, for different classes, in [12,19, 20,23,27] and [2]. The paper [1] provides an "implicit algorithm" to detect the geometricity. It seems reasonable to think that the work of M. Lustig [24] could also lead to such an algorithm, with some non-trivial additional technical work (from the same author see also [15], Section 6). Our approach here is quite different. We are not primarily interested in giving an algorithm which would be a kind of black-box, but rather in providing easy and effective characterizations of the geometric automorphisms in the class considered. In this sense, we are closer in spirit to [20]. Our work only recognizes free group automorphisms induced by pseudo-Anosov homeomorphisms without interior singularities [13]. As a counterpart to this restriction, we do not introduce highly sophisticated graph-maps, as the "improved relative train-track maps" of [1], or the "partial train-tracks with Nielsen faces" of [24]. In spite of this, let us observe that the automorphisms we deal with are not necessarily irreducible. We remind that $\alpha \in \operatorname{Aut}\left(\mathbb{F}_{n}\right)$ is reducible if $\alpha$ permutes, up to conjugation, the factors $F_{\lambda}$ of a free product decomposition of $\mathbb{F}_{n}$ of the form $\mathbb{F}_{n}=\underset{\lambda \in \Lambda}{*} F_{\lambda} * G$, and $\alpha$ is irreducible if it is not reducible. Obviously $\alpha$ is irreducible if and only if $\alpha^{-1}$ is, and this notion only depends on the class of $\alpha$ in $\operatorname{Out}\left(\mathbb{F}_{n}\right)$. We often blur the distinction between $\operatorname{Aut}\left(\mathbb{F}_{n}\right)$ and $\operatorname{Out}\left(\mathbb{F}_{n}\right)$.

In order to give a flavor of the results of this paper without entering in the details of the class of automorphisms considered, we just call them "nice". Let us now recall the definition of the growth-rate $\lambda(\alpha)$ of $\alpha \in \operatorname{Out}\left(\mathbb{F}_{n}\right)$ :

$$
\lambda(\alpha)=\sup _{C \in \mathbb{F}_{n}} \limsup _{j \rightarrow+\infty} \frac{\ln \left(\left\|\alpha^{j}(C)\right\|\right)}{j} .
$$

Here $\|w\|$ is the length of the cyclically reduced representative of $w$. We then have the following statement:

Theorem 1.1. A nice outer free group automorphism $\alpha$ is geometric if and only if $\lambda(\alpha)=\lambda\left(\alpha^{-1}\right)$. If $\alpha$ is geometric, then it is induced by a pseudo-Anosov homeomorphism without interior singularities.

We give examples of nice, non-geometric automorphisms and also of nice reducible automorphisms in Appendix D. In [20], there appears a non-geometric $\beta \in \operatorname{Out}\left(\mathbb{F}_{3}\right)$ with $\lambda(\beta)=\lambda\left(\beta^{-1}\right)$. We explain in Appendix D why this is not a nice automorphism (this is the first one considered in Example 0).

In all the papers cited above, $\alpha \in \operatorname{Out}\left(\mathbb{F}_{n}\right)$ is represented by a pair $(\psi, \Gamma), \Gamma$ being a graph with fundamental group $\mathbb{F}_{n}$ and $\psi$ a certain kind of graph-map, inducing 
$\alpha$ on the fundamental group of $\Gamma$. A feature of the work presented here is to partly use another type of representation: $\alpha \in \operatorname{Out}\left(\mathbb{F}_{n}\right)$ is represented by a pair $(K, u), K$ being a particular kind of 2-complex with fundamental group $\mathbb{F}_{n}^{\alpha}=\mathbb{F}_{n} \rtimes_{\alpha} \mathbb{Z}$ (the mapping-torus group of $\alpha)$ and $u \in C^{1}(K ; \mathbb{Z})$ is a cocycle whose cohomologyclass is associated to this semi-direct product structure after identifying $H^{1}(K ; \mathbb{Z})$ with $H^{1}\left(\mathbb{F}_{n}^{\alpha} ; \mathbb{Z}\right)$. Such a representation was introduced in [16]. Its interest lies on the fact that the 2-complexes $K$ that we consider have a particularly simple combinatorial description. They are not just mapping-tori $\Gamma \times[0,1] /(x, 1) \sim(\psi(x), 0)$ of graph-maps $(\psi, \Gamma)$ but have been "desingularized" in some sense. In particular, they are standard 2-complexes as defined in [7] (see also [25] and more recently [4]) and they are also branched surfaces (i.e. are equipped with a smooth structure) as introduced in [29] (closer in spirit, see [5]). More precisely they are "dynamical branched surfaces" as defined in [9].

The plan of the paper goes as follows: In Sections 2 and 3 the notions necessary to the statement of Theorem 3.3 are introduced. In Sections 4 and 5 we recall the basic notions about train-tracks and free group automorphisms so that we can state the "train-track version" of Theorem 3.3, this is Theorem 5.12. In Sections 6 and 7, we shed some light on the link between the above theorems. In particular, in Section 7 , there appears the process of combinatorial suspension already evoked. Sections 8 and 9 present the technical work needed to prove Theorem 3.3. The two important results are the (rather easy) Proposition 8.1 and the (harder) Proposition 9.1. In Section 10, we gather all the pieces and prove Theorems 3.3 and 5.12. Appendix A elucidates the passage from "train-tracks with circuits" to "nice train-tracks" (both were defined in Section 5). Appendix B is a brief informal discussion about the algorithmic detection of train-track with circuits among the representatives of a free group automorphism. Appendix $\mathrm{C}$ gives a characterization of the mapping-tori of pseudo-Anosov surface homeomorphisms without interior singularities. It is also proved there (Proposition C.1) that any pseudo-Anosov without interior singularities admits a train-track with circuits, and even a nice train-track when the surface is orientable and the pseudo-Anosov is orientation-preserving. Appendix D presents a bunch of examples.

\section{Standard dynamical branched surfaces}

The set of singular points in a 2-complex $K$, denoted by $\mathcal{S}(K)$, is the set of points with no neighborhood in the complex homeomorphic to a disc. A region of $K$ is a connected component of $K-\mathcal{S}(K)$.

Definition 2.1 ( $[4,7])$. A standard 2-complex is a compact 2-complex satisfying the following three properties:

(a) the set of singular points is a connected 4-valent graph with at least one vertex;

(b) each point admits a neighborhood homeomorphic to the neighborhood of some point in the interior of the cone over the 1-skeleton of the tetrahedron, see Figure 2.1;

(c) each region is a 2 -cell. 

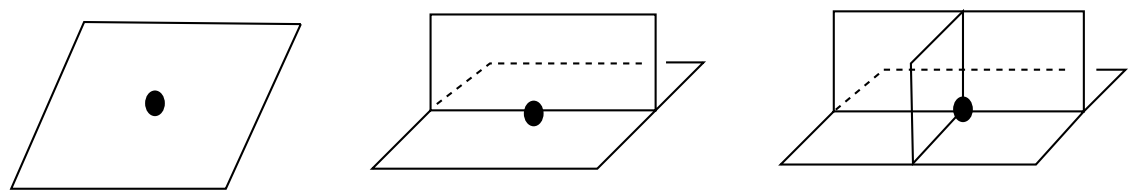

Figure 2.1. Non-singular and singular points in a standard 2-complex.

Smoothing a cellular complex $K$ consists in defining at each point of $K$ a tangent plane $T_{x} K$, which depends continuously on $x$. See Figure 2.2.

Definition 2.2. A standard branched surface is a smooth standard 2-complex.

Definition 2.3. Let $K$ be a standard 2-complex. Two oriented germs of edges at a vertex $v$ of $K$ form a source (respectively a sink) for the germ of region at $v$ that they bound if they both point away from (respectively toward) $v$.

The notion of $k$-sheeted side, $k \geq 1$, in a standard branched surface is intuitively obvious. We leave the reader elaborate the precise definition and refer him to Figure 2.2.

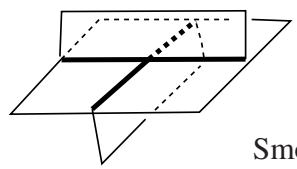

Smoothing a standard complex
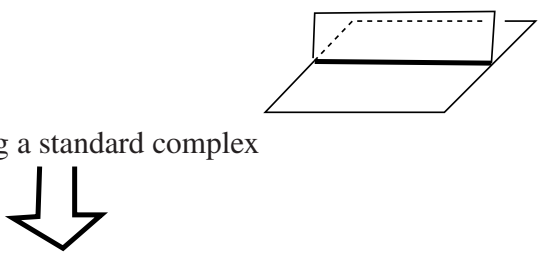

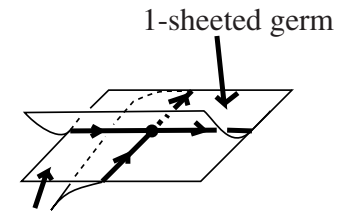

middle of the

3 -sheeted portion

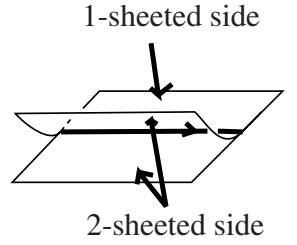

Figure 2.2. Neighborhood of the singular set in a standard (dynamical) branched surface.

Definition 2.4. A standard dynamical branched surface is a standard branched surface $\mathcal{W}$ which admits an orientation on the edges of $\mathcal{S}(\mathcal{W})$ satisfying the following properties:

(a) at each vertex $v$ of $\mathcal{W}$, the germ of region of $\mathcal{W}$ at $v$ which is 1 -sheeted (respectively in the middle of the 3 -sheeted portion) is a source (respectively a sink);

(b) the boundary of every region contains exactly one sink and one source.

Remark 2.5. The boundary of a region decomposes into two maximal positive edge-paths in the singular graph. Each one connects the source of the region to its sink. 


\section{Statement of first main theorem}

Definition 3.1. Let $\mathcal{W}$ be a standard dynamical branched surface. A positive cocycle of $\mathcal{W}$ is a cocycle in $C^{1}(\mathcal{W} ; \mathbb{Z})$ which is non-negative on the edges of $\mathcal{S}(\mathcal{W})$, and which is positive on all the positive embedded loops of $\mathcal{S}(\mathcal{W})$.

The definition below motivates the introduction of these positive cocycles.

Definition 3.2. An outer automorphism $\alpha$ of $\mathbb{F}_{n}$ is represented by a positive cocycle $u$ of a standard dynamical branched surface $\mathcal{W}$ if, after identifying the first cohomology-groups of $\mathcal{W}$ and of its fundamental group $G$, we have $\operatorname{Ker}(u)=\mathbb{F}_{n}$, $G / \operatorname{Ker}(u)=\mathbb{Z}$ and $\alpha$ is the outer automorphism of $\mathbb{F}_{n}$ associated to the short exact sequence $1 \rightarrow \operatorname{Ker}(u) \rightarrow G \rightarrow G / \operatorname{Ker}(u) \rightarrow 1$.

The subset of all the elements of $\operatorname{Out}\left(\mathbb{F}_{n}\right)$ which can be represented by positive cocycles of standard dynamical branched surfaces is denoted by $\operatorname{SDBS}\left(\mathbb{F}_{n}\right)$.

We recall further in the paper the connection between positive cocycles of standard dynamical branched surfaces and outer free group automorphisms (see [16]). We now state the first main result of this paper:

Theorem 3.3. Let $\alpha \in \operatorname{SDBS}\left(\mathbb{F}_{n}\right)$. The following properties are equivalent:

(a) $\alpha$ is geometric.

(b) If $\mathcal{W}$ is a standard dynamical branched surface which admits a positive cocycle representing $\alpha$, then $\mathcal{W}$ can be embedded in some compact 3-manifold.

(c) $\lambda(\alpha)=\lambda\left(\alpha^{-1}\right)$.

(d) $\alpha^{-1} \in \operatorname{SDBS}\left(\mathbb{F}_{n}\right)$.

\section{Train-tracks}

A graph-map is a continuous map from a graph $\Gamma$ to a graph $\Gamma^{\prime}$. We do not require that the image of a vertex by a graph-map be a vertex. We also do not require that the map be locally injective when restricted to the edges. Substituting a path in a graph by the unique locally injective path with same endpoints in the same relative homotopy-class will be called pulling-tight or reducing the path. A representative of $\alpha \in \operatorname{Out}\left(\mathbb{F}_{n}\right)$ is a pair $(\psi, \Gamma), \Gamma$ and $\psi$ being respectively a graph with fundamental group $\mathbb{F}_{n}$, and a graph-map from $\Gamma$ to itself with $\psi_{\#}=\alpha$.

A train-track (see [26] for instance) is a graph with a smooth structure, see Figure 4.1.

If $\tau$ is a train-track, we distinguish two sides at each point of $\tau$ and, in particular, if $\tau$ is trivalent, a 2-sheeted side and a 1-sheeted side at each vertex. A legal path in a train-track $\tau$ is a non-trivial locally injective path which never crosses consecutively two germs of edges in a same side of a vertex. A map $\psi$ on a trivalent $\tau$ preserves the smoothing if, for each vertex $v$ of $\tau, \psi$ sends both germs of edges in the 2-sheeted side of $v$ to a same side of $\psi(v)$, and sends the germ of edge in the 1 -sheeted side of $v$ to the other side of $\psi(v)$. 


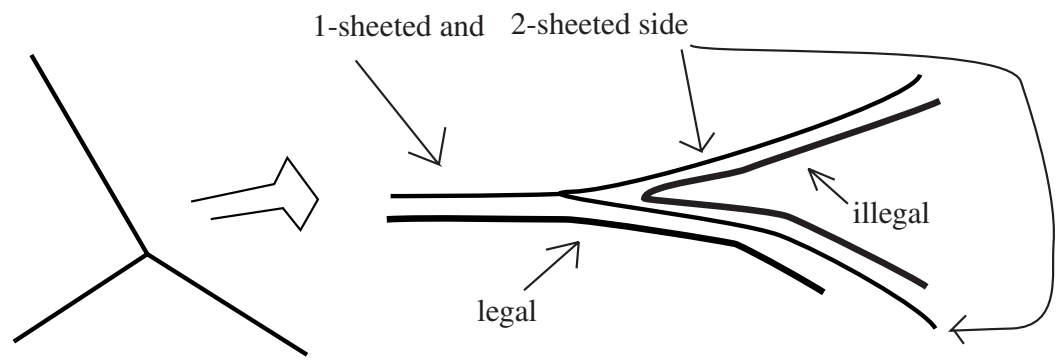

Figure 4.1. From a graph to a train-track.

Definition 4.1. A standard invariant train-track of $\alpha \in \operatorname{Out}\left(\mathbb{F}_{n}\right)$ is a representative $(\psi, \tau)$ of $\alpha$ such that $\tau$ is a trivalent train-track, and $\psi$ preserves the smoothing of $\tau$ and maps any edge to a legal path.

Example 4.2. See Figure 4.2.
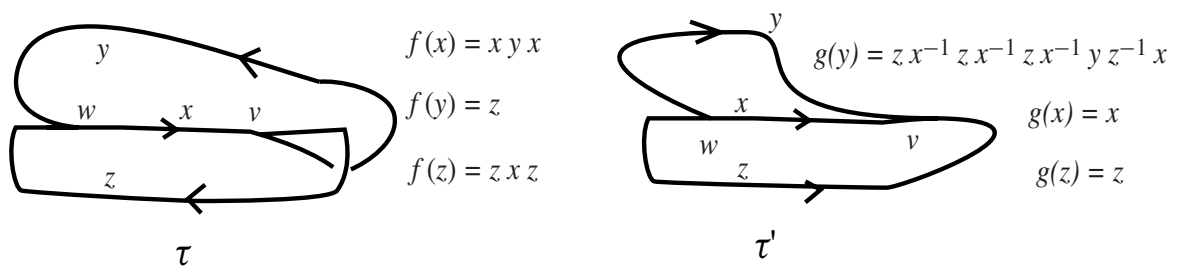

Figure 4.2. Two invariant train-tracks.

A classical consequence of the above definition is that any iterate of $\psi$ is locally injective when restricted to the edges.

\section{Train-tracks with circuits and nice train-tracks}

We begin by recalling a topological version of Stallings folds [28]:

Definition 5.1. Let $(\psi, \tau)$ be an invariant train-track.

A cancellation-pair $(p, q)$ for $(\psi, \tau)$ is a pair $p, q:[0,1] \rightarrow \tau$ of (parametrized) legal paths in $\tau$ with $p(0)=q(0)$ a vertex of $\Gamma$ and such that $\psi(p(t))=\psi(q(t))$ for any $t \in[0,1]$.

$\bar{\psi}: \bar{\tau} \rightarrow \tau$ is obtained by folding $(\psi, \tau)$ at $(p, q)$, or along $\psi(p)=\psi(q)$, if:

- $\bar{\tau}=\tau / \sim$ where $x \sim y$ if and only if $\exists t \in[0,1]$ s.t. $x=p(t)$ and $y=q(t)$;

- $\bar{\psi} \circ \pi=\psi$ with $\pi: \tau \rightarrow \bar{\tau}$ the quotient-map.

Example 5.2. In this example and the following ones of this section (which all refer to Figure 4.2), when $e$ is an oriented edge we denote by $e_{i}$ the subpath of $e$ which is mapped to the $i^{\text {th }}$ edge in the image of $e$ under the map considered. 
Two cancellation-pairs for $(f, \tau)$ respectively at $v$ and $w$ are $\left(y x_{1}, z_{1} z_{2}\right)$ and $\left(z_{3}^{-1} z_{2}^{-1}, y^{-1} x_{3}^{-1}\right)$.

For $\left(g, \tau^{\prime}\right)$ we have $\left(y_{9}^{-1} y_{8}^{-1}, x^{-1} z\right)$ and $\left(y_{1} \cdots y_{6}, z x^{-1} z x^{-1} z x^{-1}\right)$.

We denote by $\chi(\tau)=1-\operatorname{rank}\left(\pi_{1}(\tau)\right)$ the Euler characteristic of $\tau$. If $\tau$ is trivalent, then $-2 * \chi(\tau)=2 * \operatorname{rank}\left(\pi_{1}(\tau)\right)-2$ is the number of vertices in $\tau$.

Definition 5.3. Let $(\psi, \tau)$ be a standard invariant train-track. A set of cancellationpaths for $(\psi, \tau)$ is a set of $-2 \chi(\tau)$ oriented legal paths in $\tau$ such that:

(a) It is possible to fold $(\psi, \tau)$ along the cancellation-paths and get an automorphism of $\tau$.

(b) Each vertex of $\tau$ is the terminal vertex of exactly one cancellation-path.

Example 5.4. $(f, \tau)$ (respectively $\left.\left(g, \tau^{\prime}\right)\right)$ admits a set of 2 cancellation-paths: $z x$ along which one folds $z_{1} z_{2}$ with $y x_{1}$ (respectively $z x^{-1} z x^{-1} z x^{-1}$ along which one folds $y_{1} \cdots y_{6}$ with $z x^{-1} z x^{-1} z x^{-1}$ ) and $z^{-1} x^{-1}$ along which one folds $z_{3}^{-1} z_{2}^{-1}$ with $y^{-1} x_{3}^{-1}$ (respectively $x^{-1} z$ along which one folds $y_{9}^{-1} y_{8}^{-1}$ with $x^{-1} z$ ).

Definition 5.5. A train-track with circuits is a standard invariant train-track $(\psi, \tau)$ for which there exists an integer $j \geq 1$ such that $\left(\psi^{j}, \tau\right)$ admits a set $\mathcal{C}$ of cancellation-paths satisfying the following property:

for any oriented edge $e$ from $\tau$, there is an oriented subpath $s$ of $e$ and two legal paths $p_{+}, p_{-}$such that:

(a) either $s$ (respectively $s^{-1}$ ) is an oriented subpath of $p_{+}$(respectively of $p_{-}$), i.e. $p_{+}=\cdots s \cdots$ whereas $p_{-}=\cdots s^{-1} \cdots$, or $s p_{-}$is a proper oriented subpath of $p_{+}$(i.e. $\left.p_{+}=\cdots s p_{-} \cdots\right)$;

(b) $\psi\left(p_{+}\right)$and $\psi\left(p_{-}\right)$are two distinct cancellation-paths in $\mathcal{C}$.

Example 5.6. The pair $(f, \tau)$ is a train-track with circuits. Indeed $z_{2} \subset z$ and $y$ appear in the cancellation-pair $\left(z_{1} z_{2}, y x_{1}\right)$ whereas $z_{2}^{-1}$ and $y^{-1}$ appear in $\left(z_{3}^{-1} z_{2}^{-1}, y^{-1} x_{3}^{-1}\right)$. It remains to check that some subpath of $x$ also appears with its both orientations in the cancellation-pairs for some $\left(f^{j}, \tau\right)$. By looking at $f^{2}$, we get as cancellation-pairs $\left(z_{1} \cdots z_{8}, y x_{1} \cdots x_{5}\right)$ and $\left(z_{9}^{-1} \cdots z_{2}^{-1}, y^{-1} x_{7}^{-1} \cdots x_{3}^{-1}\right)$. Hence $x_{3} x_{4} x_{5} \subset x$ appears with both orientations. The pair $\left(g, \tau^{\prime}\right)$ is not a traintrack with circuits because, whatever iterate $\left(g^{j}, \tau^{\prime}\right)$ is considered, neither $y$ nor $y^{-1}$ appears as a subpath in the cancellation-pairs of $\left(g^{j}, \tau^{\prime}\right)$.

Definition 5.7. Let $(\psi, \tau)$ be a standard invariant train-track. Two cancellationpairs $\left(p_{i}, q_{i}\right),\left(p_{j}, q_{j}\right)$ of $(\psi, \tau)$ are in bad position if either $p_{i}$ or $q_{i}$ contains $p_{j}$ or $q_{j}$ as an oriented subpath (i.e. for instance $p_{i}=\cdots p_{j} \cdots$ ).

Example 5.8. The two cancellation-pairs in $\left(g, \tau^{\prime}\right)$ are in bad position. Indeed, $x^{-1} z$ which is a maximal legal path in the first one is strictly contained in $z x^{-1} z x^{-1} z x^{-1}$ which is a maximal legal path of the second one. 
Definition 5.9. A nice train-track is a train-track with circuits $(\psi, \tau)$ such that, whatever set of cancellation-paths is considered, the associated set of cancellationpairs does not contain cancellation-pairs in bad position.

The subset of all the elements of $\operatorname{Out}\left(\mathbb{F}_{n}\right)$ which admit nice train-tracks is denoted by $\operatorname{NTT}\left(\mathbb{F}_{n}\right)$.

Remark 5.10. Let $(\psi, \tau)$ be a nice train-track. Whatever set of cancellation-paths is considered, the first condition of item (a), Definition 5.5 is always satisfied.

Example 5.11. The pair $(f, \tau)$ is a nice train-track. This is in fact a representative of the pseudo-Anosov homeomorphism induced on the torus deprived of an open disc by the classical Thom automorphism $\left(\begin{array}{ll}2 & 1 \\ 1 & 1\end{array}\right)$ of $\mathbb{Z}^{2}$.

We can now state the "train-track" version of our theorem:

Theorem 5.12. Let $\alpha \in \mathrm{NTT}\left(\mathbb{F}_{n}\right)$. The following properties are equivalent:

(a) $\alpha$ is geometric.

(b) If $(\psi, \tau)$ is a nice train-track of $\alpha$, then the map $\psi$ preserves, up to homotopy, a set of reduced, unoriented loops in $\tau$ the union of which crosses exactly twice each edge of $\tau$.

(c) $\lambda(\alpha)=\lambda\left(\alpha^{-1}\right)$.

(d) $\alpha^{-1} \in \operatorname{NTT}\left(\mathbb{F}_{n}\right)$.

\section{From a positive cocycle to an outer free group automorphism}

This section is a short summary of [16].

Definition 6.1. A graph $\Gamma$ is $r$-embedded in a standard 2-complex $K$ if $\Gamma$ is embedded in $K$ transversely to $\mathcal{S}(K)$ and such that:

- The vertices of $\Gamma$ belong to the interior of the edges of $\mathcal{S}(K)$. The edges of $\Gamma$ are disjointly embedded in the regions of $K$.

- Let $v$ be a vertex of $\Gamma$ in an edge $e$ from $\mathcal{S}(K)$. There is exactly one germ of an edge of $\Gamma$ at $v$ embedded in each germ of a region of $K$ at $e$.

See Figure 6.1.

The embedding is a 2-sided embedding if $\Gamma$ admits a neighborhood in $K$ homeomorphic to the trivial I-bundle $\Gamma \times[-1,1]$.

Remark 6.2. A naïve, but useful, observation is that a graph r-embedded in a standard branched surface inherits a structure of train-track from the smooth structure of the branched surface.

Lemma 6.3. Let $K$ be a standard 2-complex. Any r-embedded, 2-sided graph in $K$ defines a cocycle in $C^{1}(K ; \mathbb{Z})$. Let $u \in C^{1}(K ; \mathbb{Z})$ be an integer cocycle of $K$. There is a unique, up to isotopy, r-embedded, 2-sided graph $\Gamma_{u}$ in $K$ representing $u$ and satisfying the following property: $(\star)$ no edge of $\Gamma_{u}$ connects two vertices in a same edge of $\mathcal{S}(K)$. 

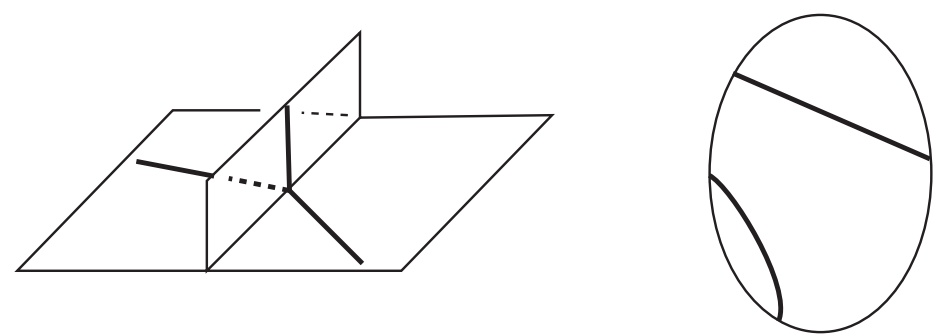

Figure 6.1. A r-embedding.

From now on all r-embedded graphs considered will satisfy property $(\star)$ above, even if not mentioned.

In the following proposition and its subsequent corollary, we describe with some details the topology of a standard dynamical branched surface with a positive cocycle. A complete proof can be found in [16].

Proposition 6.4 ( [16]). Let $\mathcal{W}$ be a standard dynamical branched surface. Then any positive cocycle $u \in C^{1}(\mathcal{W} ; \mathbb{Z})$ defines a foliation of $\mathcal{W}$ with compact graphs transversely oriented by the edges of the singular graph and such that:

(a) all the graphs are homotopically equivalent; at the exception of $n$ of them ( $n$ is the number of vertices in $\mathcal{W}$ ) all the graphs are r-embedded;

(b) the graphs which are not r-embedded contain exactly one 4-valent vertex at a vertex of $\mathcal{W}$ and otherwise satisfy all the other properties required for a $r$ embedding.

Let $\mathcal{W}$ be a standard dynamical branched surface which admits a positive cocycle. By Proposition 6.4, the points in $\mathcal{W}$ can be parametrized in coordinates $(x, \mu)$, such that $\mu \in[0,1)$ and $x$ belongs to a graph $\Gamma_{\mu}$ of the foliation given by the proposition. For any $t \geq 0$ there is a continuous deformation in $\mathcal{W}$ from $\Gamma_{\mu}$ to $\Gamma_{\mu+t-E[\mu+t]}$, where $E[\mu+t]$ denotes the greatest integer smaller than or equal to $\mu+t$. This continuous deformation induces a map $\sigma_{t}: \Gamma_{\mu} \rightarrow \Gamma_{\mu+t-E[\mu+t]}$ satisfying $\sigma_{t+t^{\prime}}=\sigma_{t} \circ \sigma_{t^{\prime}}$. This is a non-singular semi-flow. The passage from $\Gamma_{\mu}$ to $\Gamma_{\mu+t-E[\mu+t]}$ will be called pushing $\Gamma_{\mu}$ along the singular graph or along the semi-flow. Each graph $\Gamma_{\mu}$ of the foliation is a cross-section to such a semiflow, i.e. it intersects transversely, and always in the same direction, each orbit of the semi-flow. The map $\sigma_{1}: \Gamma_{\mu} \rightarrow \Gamma_{\mu}$ is the return-map of the semi-flow on its cross-section.

Corollary 6.5. Let $\mathcal{W}$ be a standard dynamical branched surface, which admits a positive cocycle $u$. With the notations above: let $t_{i}<t_{k}$ in $[0,1]$ be such that $\Gamma_{t_{i}}$ and $\Gamma_{t_{k}}$ are r-embedded.

(a) If $\Gamma_{t}$ is r-embedded for all $t \in\left[t_{i}, t_{k}\right]$, then the map from $\Gamma_{t_{i}}$ to $\Gamma_{t_{k}}$ induced by the semi-flow is homotopic to a homeomorphism. 
(b) If there is exactly one $t \in\left[t_{i}, t_{k}\right]$ such that $\Gamma_{t}$ is not $r$-embedded, then $\Gamma_{t_{k}}$ is obtained from $\Gamma_{t_{i}}$ by a Whitehead-move (see Figure 6.2) (and the map from $\Gamma_{t_{i}}$ to $\Gamma_{t_{k}}$ induced by the semi-flow is homotopic to a map induced by this move).

In particular, the return-map of the semi-flow on $\Gamma_{u}$ is homotopic to a composition of Whitehead-moves and of a homeomorphism and induces an outer automorphism on the fundamental group of the graph. This is the outer automorphism represented by $u$.

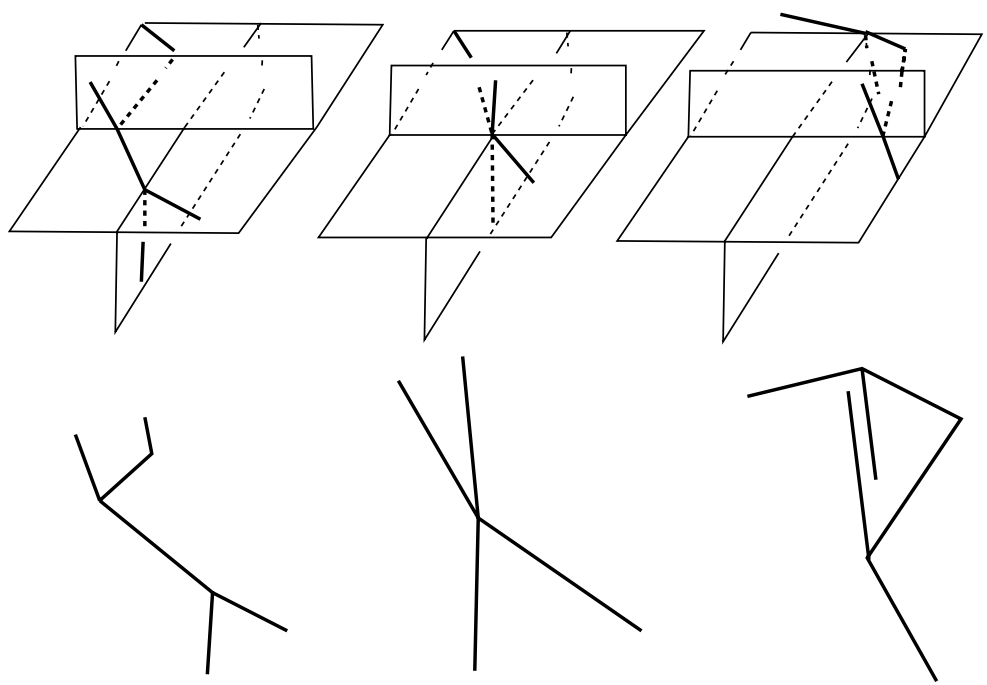

Figure 6.2. A Whitehead-move through a vertex of a dynamical branched surface.

\section{Standard dynamical branched surfaces vs nice train-tracks}

The goal of this section is to prove Theorem 7.1 below. We decompose the proof in the two Propositions 7.2 and 7.7, each one treating an inclusion.

Theorem 7.1. For any $n \geq 1, \operatorname{SDBS}\left(\mathbb{F}_{n}\right)=\operatorname{NTT}\left(\mathbb{F}_{n}\right)$.

For $n=1$ both sets are obviously empty. The proof of Proposition 7.2 below relies on a process of "combinatorial suspension", or rather of desingularization of the classical mapping-torus construction, already evoked in the introduction.

Proposition 7.2. For any $n \geq 1, \operatorname{NTT}\left(\mathbb{F}_{n}\right) \subset \operatorname{SDBS}\left(\mathbb{F}_{n}\right)$.

Let $\psi_{i}: \tau_{i} \rightarrow \tau$ be a train-track map from a train-track $\tau_{i}$ to a train-track $\tau$. Here, a train-track map is a graph-map which satisfies the properties required for the map $\psi$ of an invariant train-track $(\psi, \tau)$. 
Definition 7.3. With the notations above: a fine cancellation-pair for $\psi_{i}: \tau_{i} \rightarrow \tau$ is a cancellation-pair $\left(p_{i}, q_{i}\right)$ such that:

(a) the terminal point of neither $p_{i}$ nor $q_{i}$ is a vertex;

(b) $p_{i}^{-1} q_{i}$ is an embedded path;

(c) there is no $t_{0}$ in $[0,1]$ such that both $p_{i}\left(t_{0}\right)$ and $q_{i}\left(t_{0}\right)$ are vertices of $\tau_{i}$.

We consider a fine cancellation-pair $\left(p_{i}, q_{i}\right)$. Let $K_{\tau_{i}}=\tau_{i} \times[0,1]$. We will make the slight abuse of identifying $\tau_{i}$ with any $\tau_{i} \times\{r\}$. We define an equivalence relation $\mathcal{R}_{i}$ on $K_{\tau_{i}}$ as follows:

$(x, t) \mathcal{R}_{i}\left(x^{\prime}, t^{\prime}\right)$ if and only if $t=t^{\prime}$ and there is $t_{0} \leq t$ such that $x=p_{i}\left(t_{0}\right)$ and $x^{\prime}=q_{i}\left(t_{0}\right)$.

Definition 7.4. With the assumptions and notations above: we set $K_{i}=K_{\tau_{i}} / \mathcal{R}_{i}$. The image in $K_{i}$ of the set $\left\{\left(p_{i}(t), t\right), t \in[0,1]\right\}$ is a suspended cancellation-path.

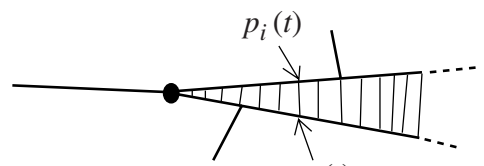

$q_{i}(t)$
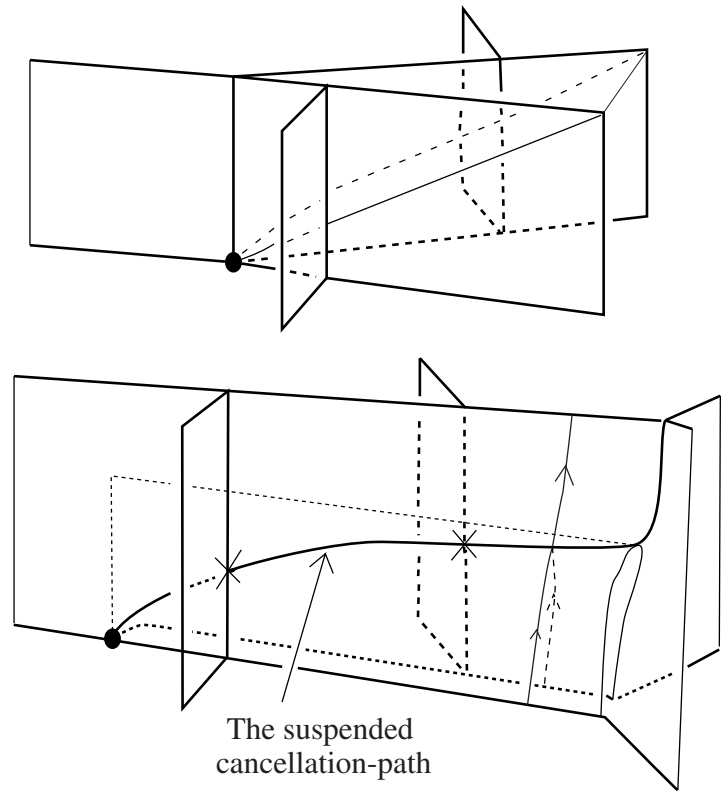

Figure 7.1. Combinatorial suspension.

The definition of a r-embedded graph in a standard 2-complex is extended in a straightforward way to the kind of 2-complex considered here.

Lemma 7.5. With the assumptions and notations above, $\mathcal{S}\left(K_{i}\right)$ is a union of intervals which can intersect each other only transversely. More precisely: 
(1) Let $v$ be any vertex of $\tau_{i}$ distinct from the vertex $p_{i}(0)=q_{i}(0)$. The singular set is formed by the suspended cancellation-path and the union of the images $I_{v}$ in $K_{i}$ of the intervals $\{v\} \times[0,1]$.

(2) All the intervals $I_{v}$ are disjoint.

(3) The suspended cancellation-path intersects an interval $I_{v}$ if and only if $v$ belongs to $p_{i}^{-1} q_{i}$.

\section{Furthermore:}

(4) The 2-complex $K_{i}$ has two boundary components, the bottom one homeomorphic to $\tau_{i}$ and the top one homeomorphic to the graph obtained by folding $\tau_{i}$ at $\left(p_{i}, q_{i}\right)$.

(5) The neighborhood of any point in (the interior of) $K_{i}$ is homeomorphic to the neighborhood of some point in (the interior of) the cone over the 1-skeleton of the tetrahedron.

(6) The image in $K_{i}$ of $\tau_{i} \times\{t\} \subset K_{\tau_{i}}$ either is a r-embedded graph, or is a graph in the interior of $K_{i}$ with one 4-valent vertex at a vertex of $\mathcal{S}\left(K_{i}\right)$ and which is otherwise r-embedded in $K_{i}$.

The notion of an almost standard branched surface and of a dynamical branched surface used in the proof below are obvious generalizations of the standard definitions introduced before. "Almost standard" for a branched surface means that there is no restriction on the topology of the regions. In a dynamical branched surface might exist regions which are open annuli or Moebius-bands but these regions have neither sources nor sinks in their boundaries. The reader will painlessly imagine the obvious adaptations to these non-standard settings of the various objects defined in the standard ones.

Proof of Proposition 7.2. By definition, a nice train-track $(\psi, \tau)$ of $\alpha \in \operatorname{Out}\left(\mathbb{F}_{n}\right)$ admits a collection of cancellation-pairs such that folding at these pairs eventually yields a diffeomorphism $h$ of $\tau$. We decompose these cancellation-pairs, by taking subpaths of the paths involved in each pair, to get an ordered sequence of fine cancellation-pairs $\left(p_{i}, q_{i}\right), i=0, \cdots, r$, in invariant train-tracks $\psi_{i}: \tau_{i} \rightarrow \tau$, which satisfies the additional property:

(d) for any $i$, neither $p_{i}$ nor $q_{i}$ contains an oriented subpath of some $p_{j}$ or $q_{j}$ with $j>i$.

The non-existence of pairs in bad position allows us to get property (d). The construction detailed before Lemma 7.5 gives a 2-complex $K_{i}$ for each $\psi_{i}: \tau_{i} \rightarrow \tau$ such that the top of $K_{i}$ can be identified via $h$ with the bottom of $K_{i+1}$. We denote by $K_{[0,1]}$ the 2 -complex resulting from these identifications. It has two boundary components: the bottom one is the bottom of $K_{0}$ and the top one is the top of $K_{r}$. They are diffeomorphic one to the other via the diffeomorphism $h$. We identify them by this diffeomorphism. 
Any point in the resulting 2-complex $\mathcal{W}$ is homeomorphic to the neighborhood of some point in the interior of the cone over the 1-skeleton of the tetrahedron. By definition, a smoothing is defined on each $\tau_{i}$, each $\psi_{i}$ (and also $h$ ) preserves this smoothing and the image of no edge crosses consecutively two germs of edges in the same side of a vertex. Thus a smoothing is defined on each $K_{i}$ and these smoothings assemble to define a smoothing on $\mathcal{W}$. Therefore $\mathcal{W}$ is an almost standard branched surface.

We equip $\mathcal{S}(\mathcal{W})$ with the orientation induced by the "from bottom to top" orientation of each $K_{i}$. Thanks to property (d), the above smoothing is compatible with this orientation (there are no "bad intersections", see Figure 7.2). By construction, the only possible types of regions in $\mathcal{W}$ are open annuli, Moebius-bands and discs. The orientation defined on $\mathcal{S}(\mathcal{W})$ is such that there is no source nor sink in the boundaries of the annuli or Moebius-bands, and exactly one source and one sink in the boundary of each disc. Thus $\mathcal{W}$ is a dynamical branched surface.

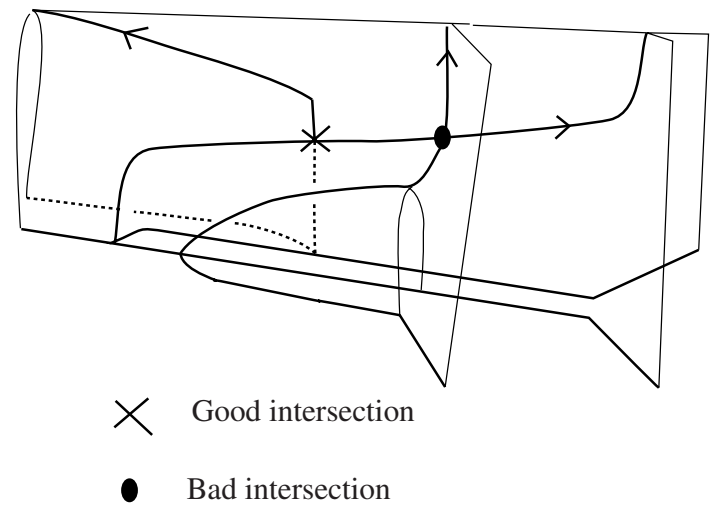

Figure 7.2. Good and bad intersections.

The images in each $K_{i}$ of the oriented intervals $\{x\} \times[0,1], x \in \tau_{i}$, glue together to define the orbits of a non-singular semi-flow which flows in a neighborhood of $\mathcal{S}(\mathcal{W})$ from the 2-sheeted side to the 1-sheeted side, when it is transverse to it. By construction, each r-embedded image of a $\tau_{i} \times\{t\}$ in $K_{i}$ defines a positive cocycle of $\mathcal{W}$. Furthermore, there is a well-defined return-map of the semi-flow on this r-embedded train-track and, by construction, this return-map induces $\alpha$ on the fundamental group. Thus $\alpha$ is represented by a positive cocycle of $\mathcal{W}$.

It only remains to check that $\mathcal{W}$ is standard, i.e. any region is a 2-cell. Assume the contrary. Consider the 2-complex $\mathcal{W}_{m}$ obtained after gluing $m \geq 1$ copies $K_{[0,1]}^{i}$ of $K_{[0,1]}$ by the identification, via $h$, of the top of $K_{[0,1]}^{i}$ to the bottom of $K_{[0,1]}^{i+1}$ for $i=1, \cdots, m$ and with $K_{[0,1]}^{m+1} \equiv K_{[0,1]}^{1}$. Following the same arguments as above, $\mathcal{W}_{m}$ is a dynamical branched surface one region of which is not a 2-cell. It obviously inherits this property from our assumption on $\mathcal{W}$. On the other hand, $\mathcal{W}_{m}$ is diffeomorphic to the dynamical branched surface obtained from $\left(\psi^{m}, \tau\right)$ by applying the combinatorial suspension process with a suitable choice 
of cancellation-paths. By Definition 5.5 and Remark 5.10, there is $j \geq 1$ such that this set of cancellation-paths satisfies that any edge of $\tau$ has a subpath which is a common subpath of two cancellation-pairs, where it appears with its two orientations. By Lemma 7.5, this implies that the combinatorial suspension process applied to $\left(\psi^{j}, \tau\right)$ yields a 2 -complex $\mathcal{W}_{j}$ whose regions are 2 -cells. We so get a contradiction for $\mathcal{W}_{j}$. Hence all regions of $\mathcal{W}$ are 2-cells.

Remark 7.6. In the course of the proof of Proposition 7.2 above, we prove in fact a slightly stronger result: if $(\psi, \tau)$ is a nice train-track representative of $\alpha$, then there is a standard dynamical branched surface $\mathcal{W}$ with positive cocycle representing $\alpha$ such that $\tau$ appears as a cross-section to a semi-flow on $\mathcal{W}$, the return-map of which is the map $\psi$.

The inclusion $\operatorname{SDBS}\left(\mathbb{F}_{n}\right) \subset \operatorname{NTT}\left(\mathbb{F}_{n}\right)$ is easier than the first one:

Proposition 7.7. Let $u$ be a positive cocycle of a standard dynamical branched surface $\mathcal{W}$. Then $u$ defines a nice train-track $\left(\psi_{u}, \tau_{u}\right)$ of the outer automorphism that it represents:

- $\tau_{u}$ is the r-embedded graph $\Gamma_{u}$ equipped with the smooth structure induced by $\mathcal{W}$;

- $\psi_{u}$ is the return-map of some semi-flow on $\mathcal{W}$ which is transverse to $\Gamma_{u}$ (see Section 6).

Proof. Consider a semi-flow on $\mathcal{W}$ which is transverse to $\tau_{u}$ and also transverse to $\mathcal{S}(\mathcal{W})$, flowing from the 2 -sheeted side to the 1 -sheeted side. Its return-map $\psi_{u}$ on $\tau_{u}$ is such that any vertex is the initial vertex of a cancellation-pair. Cut $\mathcal{W}$ along $\tau_{u}$. We get a branched surface $\mathcal{W}_{[0,1]}$ with two boundary components, both diffeomorphic to $\tau_{u}$, as in Lemma 7.5. Consider the universal covering $\widetilde{\mathcal{W}}_{[0,1]}$. This is a branched surface with two boundary components, diffeomorphic to a same smooth tree. The singular set is oriented from the bottom to the top. Consider any vertex $v$ in the bottom of $\widetilde{\mathcal{W}}_{[0,1]}$. There is an interval in $\mathcal{S}\left(\widetilde{\mathcal{W}}_{[0,1]}\right)$ from $v$ to some vertex $w$ in the top. There is also an orbit-segment of the semi-flow from $v$ to some point $u$ in the top ( $u$ is not necessarily a vertex). There is a unique reduced path in the top from $u$ to $v$. Its image under the covering-map $\pi: \widetilde{\mathcal{W}}_{[0,1]} \rightarrow \mathcal{W}_{[0,1]}$ is a cancellation-path in $\tau_{u}$ for $\psi_{u}$. All the above assertions are true for any vertex $v$ in the bottom of $\widetilde{\mathcal{W}}_{[0,1]}$. Moreover two different intervals in $\mathcal{S}\left(\mathcal{W}_{[0,1]}\right)$ which go from the bottom to the top end at two distinct vertices. Thus, we so get a set of cancellation-paths for $\left(\psi_{u}, \tau_{u}\right)$. The associated cancellation-pairs are not in bad position because the smoothing of $\mathcal{W}_{[0,1]}$ is compatible with the orientation of the singular set. Finally, the additional property required for a train-track with circuits is satisfied when suitably gluing together, bottom-to-top, a sufficiently large number of copies of $\mathcal{W}_{[0,1]}$ because, otherwise, there would be an annular or Moebius-band region. 


\section{A characterization for embedding a dynamical branched surface in a 3-manifold}

The goal of this section is to prove Proposition 8.1 below. It will be a key ingredient in the proof of Theorem 3.3. The mirror-edges appearing in the statement of this proposition are defined in the next page (Definition 8.4). A standard dynamical branched surface comes with an orientation of the edges of its singular graph. We say that this orientation and the structure of dynamical branched surface, or the smoothing, are compatible.

Proposition 8.1. Let $K$ be a standard 2-complex. Assume that some orientation of $\mathcal{S}(K)$ is compatible with a structure of dynamical branched surface $\mathcal{W}$. The following three properties are equivalent:

(a) $\mathcal{W}$ admits an embedding in a compact 3-manifold.

(b) At each vertex of $\mathcal{W}$, either both outgoing edges are mirror-edges or none of them is.

(c) The reverse orientation on the edges of $\mathcal{S}(K)$ is compatible with a structure of dynamical branched surface on $K$.

Let $\mathcal{W}$ be a standard branched surface. As was already illustrated by many figures, like Figure 8.1, one can always choose the smooth embedding in $\mathbb{R}^{3}$ of a neighborhood of a vertex in $\mathcal{W}$ so that there are four germs of regions contained in the horizontal plane of $\mathbb{R}^{3}$ and two outside. Up to isotopy, these are two types of such smooth embedding, see Figure 8.1, which differ by the cyclic ordering of the germs of regions around the edges.

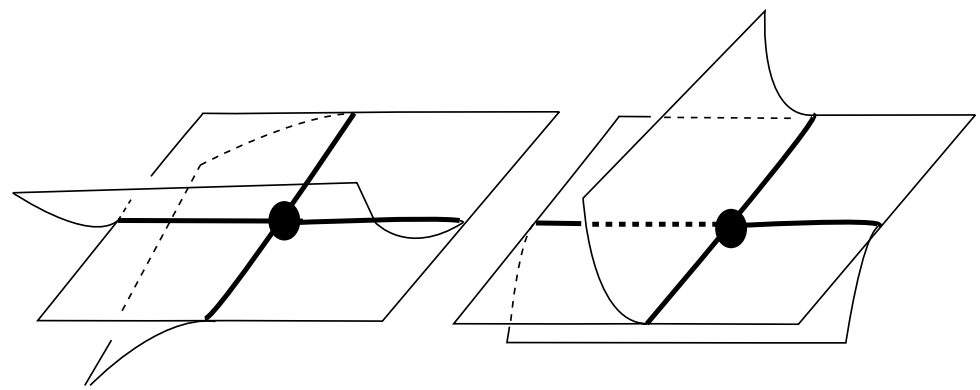

Figure 8.1. Two distinct ways to embed a neighborhood of a vertex.

Remark 8.2. When considering a standard dynamical branched surface $\mathcal{W}$, it is always possible to choose the above local $\mathbb{R}^{3}$-embeddings of $\mathcal{W}$ so that all have the same type. This is a consequence of the fact that the singular set $\mathcal{S}(\mathcal{W})$ admits an orientation compatible with the smoothing, but this is false when considering an arbitrary branched surface. This observation is important to the understanding of the so-called "mirror-edges". We advise the reader to choose once and for all his preferred type. 
A smooth structure in a neighborhood of a vertex of the singular set of a standard 2-complex (in particular a structure of branched surface) defines a cyclic ordering on the germs of edges at this vertex, unique up to reversal. We will sometimes say that these cyclic orderings and the given local smooth structure are compatible.

Definition 8.3. A circuit in a standard branched surface $\mathcal{W}$ is an immersed loop $\mathcal{C}$ of the singular graph such that if $g, g^{\prime}$ are any two germs of edges at a vertex $v$ in $\mathcal{W}$ which are consecutive in $\mathcal{C}$, then $g, g^{\prime}$ are not consecutive with respect to the cyclic ordering on the germs of edges at $v$ defined by the structure of branched surface.

Definition 8.4. An edge $e$ from the singular graph of a standard dynamical branched surface $\mathcal{W}$ is a mirror-edge if, for any region $R$ of $\mathcal{W}$, for any maximal positive path $p$ in $\partial R$, then either $p$ does not contain $e$ or $p$ contains $e$ in first, in second or in last position.

In Lemma 8.5 below, we gather some easy observations about mirror-edges. The point (a) comes from [17]. For points (b) and (c), we refer the reader to Figures 8.2 and 8.3 .

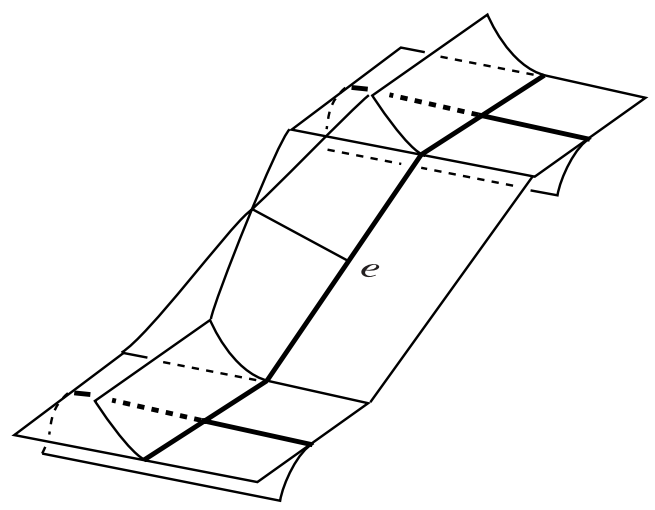

Figure 8.2. Mirror-edge.

Lemma 8.5. Let $\mathcal{W}$ be a standard dynamical branched surface. Then:

(a) Any circuit of $\mathcal{W}$ contains at least one mirror-edge.

(b) If $e_{1} e_{2} \cdots e_{k}, k \geq 2$, is a maximal positive edge-path in the boundary of a region of $\mathcal{W}$, then either $k=2$ and $e_{2}$ is not a mirror-edge or both $e_{2}$ and $e_{k}$ are mirror-edges and these are the only one in $e_{2} \cdots e_{k}$.

(c) An edge e from $\mathcal{S}(\mathcal{W})$ is a mirror-edge if and only if no smooth $\mathbb{R}^{3}$-embedding of a neighborhood of e in $\mathcal{W}$ defines two $\mathbb{R}^{3}$-embeddings of the same type when restricted to neighborhoods of the vertices of $e$.

The point (c) of Lemma 8.5 together with the criterion for embedding a standard 2-complex in a 3-manifold given by [4] lead to: 
Lemma 8.6. A standard dynamical branched surface $\mathcal{W}$ can be embedded in a 3manifold if and only if there is an even number of mirrors in the boundary of any region of $\mathcal{W}$.

For counting the number of "mirrors in the boundary of a region $R$ ", we count the number of times we pass over a mirror-edge when reading the closed edge-path $\partial R$, starting at any vertex of $\partial R$.

Lemma 8.7 ( [17]). With the notations and assumptions of Proposition 8.1, the reverse orientation on the edges of $\mathcal{S}(K)$ is compatible with a structure of dynamical branched surface if and only if any edge of $\mathcal{S}(\mathcal{W})$ appears exactly once in one-tolast position along all the maximal positive paths in the boundaries of the regions of $\mathcal{W}$.

Lemma 8.8. With the notations and assumptions of Proposition 8.1, the reverse orientation on the edges of $\mathcal{S}(K)$ is compatible with a structure of dynamical branched surface if and only if, at each vertex of $\mathcal{W}$ either both outgoing edges are mirror-edges or none of them is.

Proof. Assume that some vertex $v$ of $\mathcal{W}$ admits two outgoing edges of distinct types. Let $e$ be the non-mirror outgoing edge. Then the incoming edge at $v$ which precedes $e$ along its circuit never appears in one-to-last position in a maximal positive path of the boundary of a region. For the converse, let $v$ be any vertex of $\mathcal{W}$ and let $e$ be an incoming edge at $v$. Let $f$ (respectively $g$ ) be the outgoing edge at $v$ which follows (respectively which does not follow) $e$ in its circuit. From Figure 8.3:

- if both $f$ and $g$ are mirror-edges, then $e$ appears in one-to-last position in a maximal positive path of the boundary of the region which consecutively contains $e$ and $f$;

- if neither $f$ nor $g$ are mirror-edges, then $e$ appears in one-to-last position in a maximal positive path of the boundary of the region which consecutively contains $e$ and $g$.

Lemma 8.8 follows.

Proof of Proposition 8.1. The equivalence between (b) and (c) is the content of Lemma 8.8. Let us prove $(a) \Leftrightarrow(b)$. By Lemma 8.6, $\mathcal{W}$ cannot be embedded in a 3-manifold if and only if some region $R$ admits an odd number of mirrors in its boundary. We denote by $f$ and $g$ the outgoing edges at the source $s$ of $R$. By item (b) of Lemma 8.5, there is an even number of mirrors in the subpath of $\partial R$ containing the sink of $R$ and which goes from the terminal vertex of $f$ to the terminal vertex of $g$. Thus the total number of mirrors in $\partial R$ is odd if and only if there is an odd number of mirrors in $g^{-1} f$, whence the conclusion. 


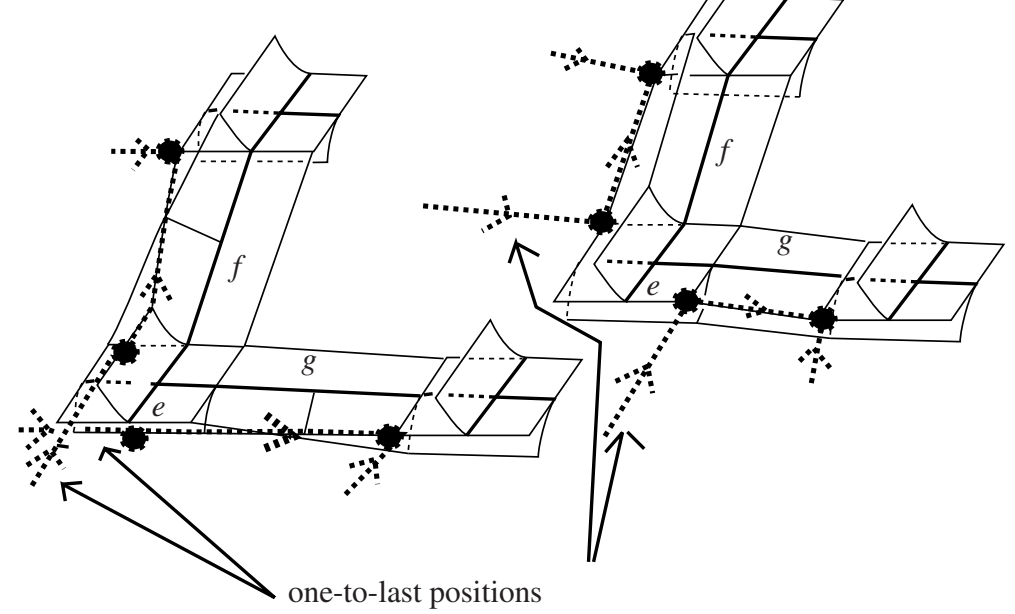

Figure 8.3. When the two outgoing edges share the same type.

\section{From non-embeddability to non-geometricity}

Here the goal is to prove the following result:

Proposition 9.1. Let $\alpha \in \operatorname{SDBS}\left(\mathbb{F}_{n}\right)$ be represented by a positive cocycle of a standard dynamical branched surface $\mathcal{W}$. If $\mathcal{W}$ cannot be embedded in a compact 3-manifold, then $\lambda\left(\alpha^{-1}\right)<\lambda(\alpha)$.

The first step is to simplify the combinatorics of the branched surface. In each region, there is a line connecting the source of the region to its sink. The union of these lines form a union of disjoint loops embedded in $\mathcal{W}$, termed cycles.

Lemma 9.2. Let $\alpha \in \operatorname{SDBS}\left(\mathbb{F}_{n}\right)$ be represented by a positive cocycle of a standard dynamical branched surface $\mathcal{W}$ which does not embed in a 3-manifold. There is $k \geq 1$ and a standard dynamical branched surface $\mathcal{V}$ admitting a positive cocycle $u$ representing $\alpha^{k}$ such that:

- The r-embedded graph $\Gamma_{u}$ intersects each cycle and each circuit of $\mathcal{V}$ exactly once. In particular, there are at least two circuits and two cycles in $\mathcal{V}$ and the circuits have no self-intersection.

- $\mathcal{V}$ does not embed in a 3-manifold.

Proof. We cut $\mathcal{W}$ along a r-embedded graph associated to a positive cocycle representing $\alpha$. Gluing in a suitable way a sufficiently hight number of copies of the resulting 2-complex, and then identifying its top and bottom, yields a standard dynamical branched surface $\mathcal{V}$ as announced. 
From now on, $\mathcal{V}$ and $u$ are assumed to satisfy the conclusions of Lemma 9.2.

The second step is to give an explicit definition of a map "induced" by a positive cocycle and representing the associated outer free group automorphism.

Let $\Gamma_{u}$ be the r-embedded graph associated to the positive cocycle $u \in C^{1}(\mathcal{V} ; \mathbb{Z})$. It defines a sequence of positive cocycles $u_{i}, i=0, \cdots, n$, with $u=u_{0}=u_{n}$, and of associated r-embedded graphs $\Gamma_{u_{i}}$ such that $\Gamma_{u_{i}}$ is obtained from $\Gamma_{u_{i-1}}$ by a Whitehead-move. This Whitehead-move corresponds to the passage through a vertex of $\mathcal{V}$. We define in Figure 9.1 an elementary-map, that is the map associated to such a passage.

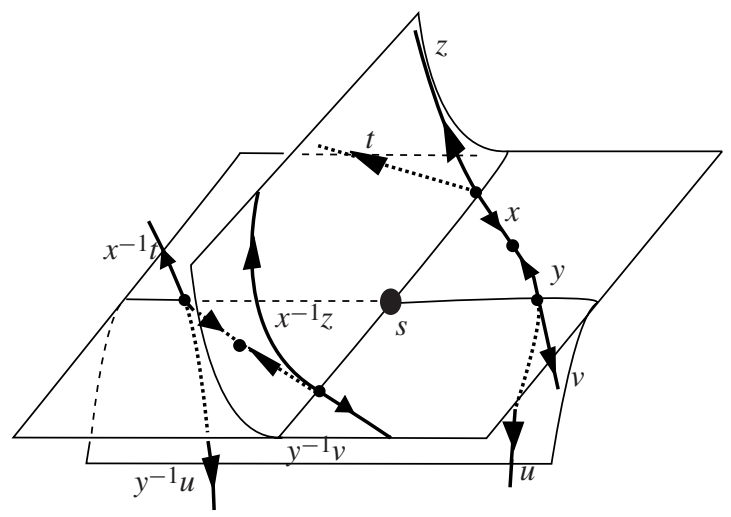

Figure 9.1. The elementary-map.

Let us describe more this figure. We subdivide the edges of the $\Gamma_{u_{i}}$ 's at the intersection points with the cycles. With respect to the orientation of the edges of $\mathcal{S}(\mathcal{V})$, $\Gamma_{u_{i}}$ is just after the vertex $s$ of $\mathcal{V}$, whereas $\Gamma_{u_{i-1}}$ is just before $s$.

The map, denoted by $\psi_{i}$, is defined from $\Gamma_{u_{i-1}}$ to $\Gamma_{u_{i}}$ by the labels put on the edges of $\Gamma_{u_{i-1}}$. No label means that the edge is collapsed. Outside the edges contained in the small contractible neighborhood of $s \in \mathcal{V}$ drawn in Figure 9.1, there is a trivial identification between the edges from $\Gamma_{u_{i}}$ and from $\Gamma_{u_{i-1}}$. The edge from $\Gamma_{u_{i-1}}$ which belongs to the germ of region in the middle of the 3 -sheeted portion is collapsed. The other edges from $\Gamma_{u_{i-1}}$ in this figure are dilated through an half of the region with source $s$, two covering $x^{-1}$ and the other two $y^{-1}$.

We feel important to stress that, because of the "symmetry" in the definition of an elementary-map, such a map does not depend on the way the complex has been smoothed in a neighborhood of the vertices (as long as the local smoothing is compatible with the orientation of the edges of the singular set).

We let $\psi_{u}$ be the graph-map on $\Gamma_{u}$ defined by $\psi_{u}=\psi_{n} \circ \psi_{n-1} \circ \cdots \circ \psi_{1}$. Observe that the 2-valent vertices of $\Gamma_{u}$ are periodic orbits (in fact fixed points because $\mathcal{V}$ and $\Gamma_{u}$ satisfy the conclusions of Lemma 9.2) for $\psi_{u}$. In other words, the cycles are periodic orbits for the semi-flow on $\mathcal{V}$ admitting $\psi_{u}$ on $\Gamma_{u}$ as returnmap. This semi-flow is not transverse everywhere to the singular set.

The following lemma is obvious from the definitions. 
Lemma 9.3. With the notations above, up to collapsing the edges of $\Gamma_{u}$ with trivial images under $\psi_{u},\left(\psi_{u}, \Gamma_{u}\right)$ is an invariant train-track of the outer free group automorphism defined by $u$ ( $\Gamma_{u}$ is equipped with the train-track structure inherited from the smooth structure of $\mathcal{V})$.

We revert the orientation of all the edges. The cocycle $-u$ is a positive cocycle of the resulting standard dynamical 2-complex, denoted by $\mathcal{D}$. If $\beta$ is the outer automorphism represented by $u$, the outer automorphism represented by $-u$ is $\beta^{-1}$. In the same way as in Lemma 9.3, we define a representative $\left(\psi_{-u}, \Gamma_{-u}\right)$ of $\beta^{-1}$.

The incidence matrix $M=\left(m_{i j}\right)$ of a graph-map $f$ mapping vertices to vertices is defined by setting $m_{i j}$ equal to the number of times the edge $e_{j}^{ \pm 1}$ appears in $f\left(e_{i}\right)$. We denote by $\lambda(f)$ or $\lambda(M)$ the growth-rate of the map $f$, which is the greatest positive eigenvalue of the matrix $M$.

Lemma 9.4. For any $k \geq 1$, the volume (i.e. the sum of the entries) of the incidence matrix $M_{u}^{k}$ of $\psi_{u}^{k}$ is equal to the volume of the incidence matrix $M_{-u}^{k}$ of $\psi_{-u}^{k}$.

Recall the construction of the graph $G(M)$ associated to the incidence matrix $M=\left(m_{i j}\right)$ of a graph-map mapping vertices to vertices: the vertices $v_{i}$ of $G(M)$ are in bijection with the edges $e_{i}$ of the graph; there are $m_{i j}$ oriented edges going from $v_{i}$ to $v_{j}$. The volume of $M$ is equal to the number of outgoing edges in $G(M)$.

Proof. Consider the cellular decomposition of $\mathcal{V}$ obtained by subdividing each region by an edge connecting the source to the sink. Take the graph dual to this cellular decomposition. We delete the edges dual to the 1-cells not in the singular graph (i.e. the 1-cells dual to the edges of the cycles). We collapse each edge $e$ which has a vertex in an edge from $\mathcal{S}(\mathcal{V})$ which is outgoing at the source of the region containing $e$. We orient each edge $e$ of the resulting graph so that its initial vertex belongs to an edge from $\mathcal{S}(\mathcal{V})$ which is outgoing at the source of the region containing $e$ (this means, roughly speaking, that the edges are oriented according to the orientation of the semi-flow). See Figure 9.2.

We denote by $\widetilde{G}_{u}$ the graph so constructed. The same construction applied to $\mathcal{D}$ (i.e. after reversing the orientation of the edges of $\mathcal{S}(\mathcal{V})$ ) yields a graph denoted by $\widetilde{G}_{-u}$. The construction of $\widetilde{G}_{u}$, and of $\widetilde{G}_{-u}$, can be realized embedded in the 2-complex. By construction also, any positive loop in $\widetilde{G}_{u}$ defines a periodic orbit of $\psi_{u}$, and conversely any periodic orbit of $\psi_{u}$ defines a positive loop in $\widetilde{G}_{u}$. The same conclusions hold for $\widetilde{G}_{-u}$.

There is an isomorphism $\mu: \widetilde{G}_{u} \rightarrow \widetilde{G}_{-u}$ which reverses the orientation of the unique edge of $\widetilde{G}_{u}$ in each 2-cell which connects the edge of the singular graph originating at the source to the edge ending $\underset{\sim}{\text { at }}$ the sink. The isomorphism $\mu$ preserves the orientation of the other edges. Let $\widetilde{R}$ denote the union of the edges of $\widetilde{G}_{u}$ the orientation of which is reversed under $\mu$. The subgraph $\widetilde{R}$ is a union of disjoint embedded loops, positively oriented, and homotopic to positive loops of the singular graph. In Figure 9.2, one of these loops is represented in dash. Among the edges of $R$, we distinguish $\mathcal{R}_{0}$, the set of edges which do not intersect $\Gamma_{u}$ in an essential way (i.e. such that the intersection point can be suppressed by a small homotopy 

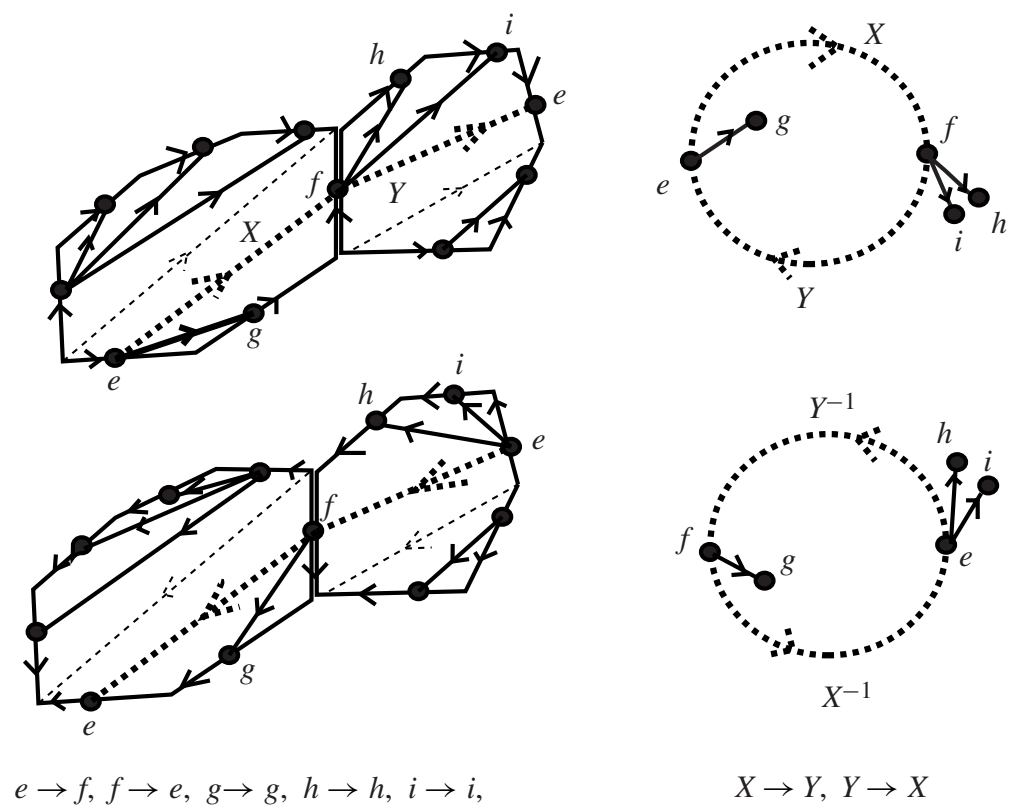

Figure 9.2. The graphs $\widetilde{G}_{u}$ and $\widetilde{G}_{-u}$.

of $\Gamma_{u}$ ). Since $\Gamma_{u}$ intersects any positive loop of the singular graph, this set $\mathcal{R}_{0}$ is a forest in $\mathcal{R}$. We collapse $\mathcal{R}_{0}$. The resulting graph is isomorphic to $G\left(M_{u}\right)$ by an orientation-preserving isomorphism. By collapsing $\mu\left(\mathcal{R}_{0}\right) \subset \widetilde{G}_{-u}$, we similarly get $G\left(M_{-u}\right)$.

If $\pi_{u}$ and $\pi_{-u}$ are the collapsing maps, $\mu$ induces an isomorphism $\bar{\mu}: G\left(M_{u}\right) \rightarrow$ $G\left(M_{-u}\right)$ which reverses the orientations of the edges of $\pi_{u}(\mathcal{R})$ and preserves the orientation of the other edges. From our description of $\mathcal{R}$ and of $\mathcal{R}_{0}, \pi_{u}(\mathcal{R})$ is a union of positive loops such that the number of incoming edges at a given vertex is equal to the number of outgoing edges. Thus the number of outgoing edges of $\pi_{-u}(\mu(\mathcal{R}))=\bar{\mu}\left(\pi_{u}(\mathcal{R})\right)$ is equal to the number of outgoing edges of $\pi_{u}(\mathcal{R})$. Therefore, $G\left(M_{u}\right)$ and $G\left(M_{-u}\right)$ admit the same number of outgoing edges, so that the volumes of $M_{u}$ and $M_{-u}$ are equal.

To conclude for the powers $M_{u}^{k}$ and $M_{-u}^{k}$, just consider the standard dynamical 2-complexes constructed for $\psi_{u}^{k}$ and $\psi_{-u}^{k}$ as was suggested in the proof of Lemma 9.2 and apply the same arguments as above.

Lemma 9.5. With the notation and assumptions above:

(a) Let e be an edge of $\Gamma_{u}$ and let $R$ be the region which contains $e . \psi_{u}(e)$ is trivial if and only if there is no vertex from $\mathcal{S}(\mathcal{V})$ in the positive path in $\partial R$ from the trivalent vertex of e to the sink of $R$. 
(b) Let $p$ be a legal edge-path in $\Gamma_{u}$. Then $\psi_{u}(p)=p$ if and only if the endpoints of $p$ are 2-valent vertices and neither $p$ nor any image of $p$ along the semiflow intersects a mirror-edge. In particular the boundary of any region entirely crossed by $p$ or any of its images contains exactly four edges of $\mathcal{S}(\mathcal{V})$.

(c) There are $j \geq 1$ and $M>0$ such that, if $p$ is a legal edge-path in $\Gamma_{u}$ with length greater than $M$, then $\Gamma_{u}=\psi^{j}(p)$. The same conclusion holds if $p$ is any legal loop in $\Gamma_{u}$.

(d) There is $j \geq 1$ such that, if some image under the semi-flow of an edge e from $\Gamma_{u}$ intersects a mirror-edge, then $\Gamma_{u}=\psi^{j}(e)$.

(e) If $e$ and $f$ are the two edges in the 2-sheeted side of a trivalent vertex of $\Gamma_{u}$, then $\Gamma_{u}=\psi^{j}(e)$ or $\Gamma_{u}=\psi^{j}(f)$.

The first item is obvious from the definition of $\psi_{u}$. We refer the reader to Figure 9.3 to see the phenomenon of dilation crucial for understanding the other items. The second item is easily deduced from this figure.

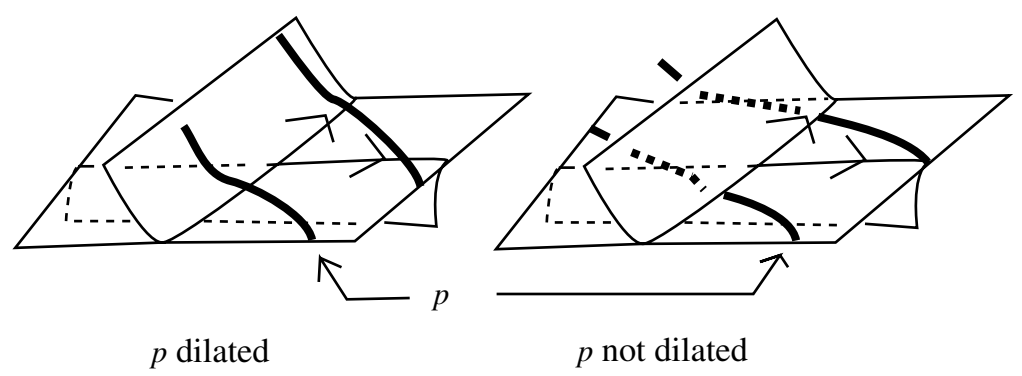

Figure 9.3.

The third item was proven in [17]. It comes from the fact that any circuit contains a mirror-edge (see Lemma 8.5, item (a)). The last assertions are consequences of this one.

The 2-complex $\mathcal{D}$ can be smoothed in a small neighborhood of each vertex, such that the smooth structure is compatible with the orientation of the singular graph. Notice however that the 2-complex $\mathcal{D}$ does not necessarily admit such a smooth structure along the whole singular graph. The following lemma strengthens, in some sense, Proposition 8.1.

Lemma 9.6. With the notations above, let $\mathcal{M}$ be the set of open edges e from $\mathcal{S}(\mathcal{V})$ such that e is a mirror-edge of $\mathcal{V}$ whereas the other edge of $\mathcal{S}(\mathcal{V})$ with same initial vertex is not. Then $\mathcal{D}$ can be smoothed along the complement of $\mathcal{M}$ in $\mathcal{S}(D)$ in a way compatible with the orientation of $\mathcal{S}(\mathcal{D})$.

Proof. The lemma relies upon the two following observations:

- Keep the same cyclic orderings for $\mathcal{D}$ as for $\mathcal{V}$ on the germs of edges at each vertex. Then $\mathcal{D}$ can be smoothed along an edge $e$ in a way compatible with the orientation of $\mathcal{S}(\mathcal{D})$ and with these cyclic orderings if and only if $e$ is not a mirror-edge of $\mathcal{V}$. See Figure 9.4. 


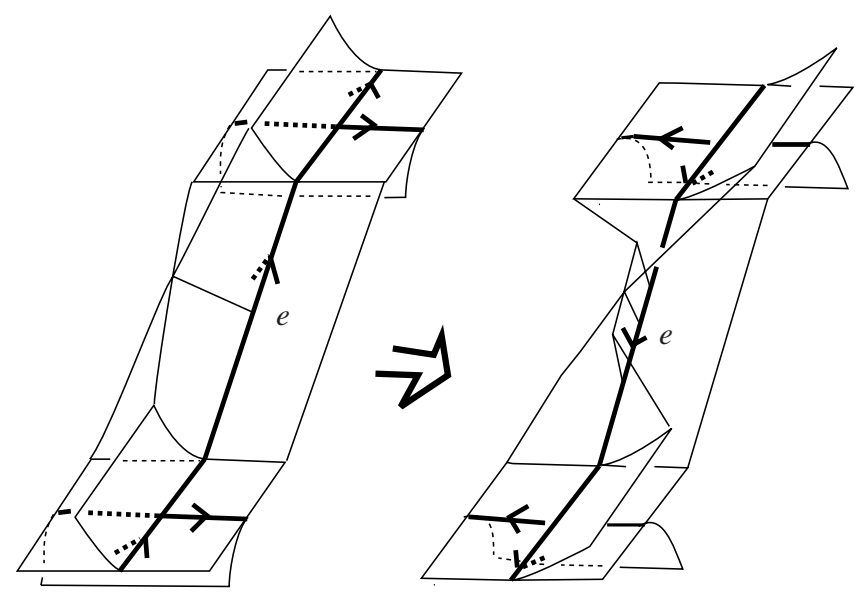

Figure 9.4. Reversing the orientation of a mirror-edge.

- Permute the two incoming, or the two outgoing, edges of $\mathcal{D}$ at some vertex $v$. Let $e$ be an incoming edge at $v$. If $\mathcal{D}$ was smoothable (respectively non-smoothable) along $e$, in a compatible way, before the change of cyclic ordering, then $\mathcal{D}$ is not smoothable (respectively smoothable) along $e$ in a compatible way after the permutation. Nothing changes for the other edges of the singular graph. We leave the reader check by inspection.

To obtain the lemma, it then suffices to permute each pair of mirror-edges in $\mathcal{V}$ which have a same initial vertex, reverse the orientation of the edges and then smooth in a neighborhood of each vertex in a way compatible with both the orientation and the cyclic orderings on the germs of edges. These local smoothings can be extended along the singular graph as announced in the lemma.

Lemma 9.7. With the notations above, let $B_{u}$ (respectively $B_{-u}$ ) be the maximal, not necessarily connected, proper subgraph of $\Gamma_{u}$ which is invariant under $\psi_{u}$ (respectively under $\psi_{-u}$ ). Then $B_{u}$ is a union of disjoint, legal embedded intervals (in particular it is a forest) and $\lambda\left(\psi_{u \mid B_{u}}\right)=\lambda\left(\psi_{-u \mid B_{-u}}\right)=1$. Moreover, if $\pi_{u}: \Gamma_{u} \rightarrow \bar{\Gamma}_{u}$ (respectively $\pi_{-u}: \Gamma_{-u} \rightarrow \bar{\Gamma}_{-u}$ ) is the map collapsing the edges of $\Gamma_{u}$ with trivial images under $\psi_{u}$ (respectively under $\left.\psi_{-u}\right)$, then $\pi_{u}\left(B_{u}\right)=$ $\pi_{-u}\left(B_{-u}\right)$.

Proof. The point (c) of Lemma 9.5 tells us that any legal path in $B_{u}$ is an interval. The point (e) in the same lemma tells us that $B_{u}$ contains no illegal turn. Thus the connected components of $B_{u}$ are legal intervals. It is straightforward that the growth-rate of the map restricted to $B_{u}$ is 1 .

Let $p$ be a $\psi_{u}$-invariant legal edge-path. By the point (b) of Lemma 9.5, and by definition of the elementary-maps, it readily follows that $p$ is $\psi-u$-invariant. Let us now consider an edge $e$ of $\Gamma_{-u}$ outside $B_{u}$ such that $\psi_{-u}(e)$ is non-trivial. As 
long as the image of $e$ under the semi-flow remains a legal edge-path, the same arguments as for Lemma 9.5 apply, so that the image of $e$ is a legal edge-path which eventually covers all the graph if $\mathcal{D}$ is smooth. Otherwise, at some point, the image of $e$ decomposes into two legal edge-paths. Since we do not reduce the image, iterating the process yields a concatenation of an increasing number of legal edge-paths which covers the graph. We so get the lemma.

Lemma 9.8. Assume that $\mathcal{D}$ does not admit a smoothing compatible with the orientation of its singular set. There is $m \geq 1$, an invariant train-track $\left(\psi_{m}, \tau\right)$ representing the same automorphism as $\left(\psi_{u}^{m}, \Gamma_{u}\right)$ and a representative $\left(\phi_{m}, \tau\right)$ of the same automorphism as $\left(\psi_{-u}^{m}, \Gamma_{-u}\right)$ with $\lambda\left(\phi_{m}\right)<\lambda\left(\psi_{m}\right)$.

Proof. By Proposition 8.1, some vertex in $\mathcal{V}$ is the initial vertex of both a mirroredge and a non-mirror-edge. We consider the partial smooth structure given by Lemma 9.6: $\mathcal{D}$ is smooth except along a non-empty set $\mathcal{M}$ of open edges in $\mathcal{S}(\mathcal{D})$, which does not contain two edges with same terminal vertex.

Let us consider an edge $f_{0}$ from $\mathcal{M}$. Consider the edge $f_{1}$ of $\mathcal{S}(\mathcal{D})$ which is outgoing at $t\left(f_{0}\right)$ and does not follow $f_{0}$ in its circuit. Without loss of generality, we can assume that $\Gamma_{-u}$ connects the two outgoing edges at $t\left(f_{0}\right)$ in the region admitting $t\left(f_{0}\right)$ as a source. We embed $\Gamma_{-u}$ in $\mathcal{D}$ so that each vertex of $\Gamma_{-u}$ belongs to a small neighborhood of the initial vertex of the edge from $\mathcal{S}(\mathcal{D})$ containing it. We equip $\Gamma_{-u}$ with the induced smooth structure.

Let $\Gamma_{-u_{1}}$ be the r-embedded graph connecting the two incoming edges at $t\left(f_{0}\right)$ in the region which admits $t\left(f_{0}\right)$ as a sink. We assume that the two trivalent vertices $v_{1}, v_{2}$ of $\Gamma_{-u_{1}}$ in these incoming edges belong to a small neighborhood of $t\left(f_{0}\right)$ and $\Gamma_{-u_{1}}$ is equipped with the smooth structure induced by this embedding.

Let $v_{1}$ be the vertex of $\Gamma_{-u_{1}}$ in $f_{0}$. Since no vertex from $\mathcal{S}(\mathcal{D})$ is the terminal vertex of two edges in $\mathcal{M}$, some image $\psi_{-u}(y)$ of an edge $y$ crosses the illegal legal turn of $\Gamma_{-u_{1}}$ at $v_{1}$ and consecutively crosses the legal turn at $v_{2}$. See Figure 9.5. Pushing $\Gamma_{-u_{1}}$ through $t_{0}$ then creates a cancellation.

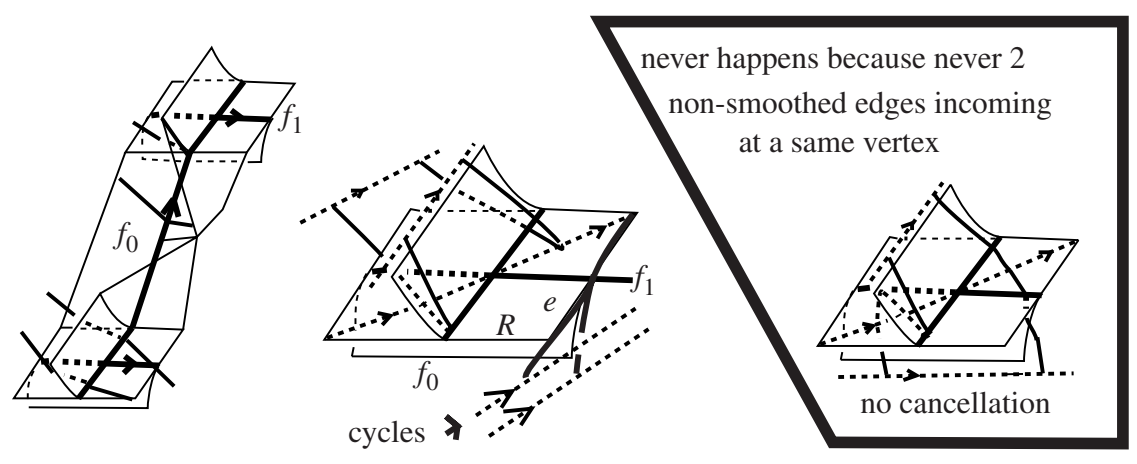

Figure 9.5. How cancellations arise.

Let $v_{0}$ be the vertex of $\Gamma_{-u}$ in $f_{1}$. Consider the turn $\tau$ of $\Gamma_{-u}$ at $v$ formed by the edge in the 1 -sheeted side and the edge $e$ in the region $R$ containing the turn 
$f_{0} f_{1}$. The legal turn of $\Gamma_{-u_{1}}$ at $v_{1}$ which has one edge in $R$ is mapped to $\tau$ by the passage through $t\left(f_{0}\right)$. Since $f_{0}$ is in $\mathcal{M}$, by pulling $\Gamma_{-u_{1}}$ along $f_{0}$ until a small neighborhood of its initial vertex, this turns becomes illegal. By definition, no illegal turn is in the image of an elementary-map when pushing through a vertex of the singular set (this is only after pushing along an edge that an illegal turn may appear). On the contrary, any legal turn which belongs to a small neighborhood of the vertex though which we push appears twice. Observe that $\tau$ is also a legal turn for $\Gamma_{u}$, and that the observations of the last sentence hold for $\mathcal{V}$. It follows that the turn $\tau$ of $\Gamma_{-u}$ appears a smaller number of times in the image of $\psi_{-u}$ than in the image of $\psi_{u}$. Moreover, since $f_{0}$ is in $\mathcal{M}$, the edge $e$ of $\Gamma_{-u}$ introduced above is outside $B_{u}$, so that $\tau$ is not in $B_{u}$. Thus the number of times $\tau$ appears in the image of $\psi_{u}^{k}$ grows exponentially faster with $k \rightarrow \infty$ than the number of times $\tau$ appears in the image of $\psi_{-u}^{k}$.

We have a natural one-to-one correspondance between the turns of $\Gamma_{u}$ and those of $\Gamma_{-u}$ (equipped with the smooth structure defined at the beginning of this proof) such that any legal turn $\tau^{\prime}$ of $\Gamma_{-u}$ corresponds to a legal turn of $\Gamma_{u}$ which is crossed at least the same number of times by the image of $\Gamma_{u}$ under $\psi_{u}$ than $\tau^{\prime}$ is crossed by the image of $\Gamma_{-u}$ under $\psi_{-u}$. Moreover, no illegal turn of $\Gamma_{-u}$ is crossed by the image of some edge under $\psi_{-u}$.

Therefore, by Lemma 9.4, after collapsing $B_{u}$, and by pulling-tight each iterate of $\psi_{-u}$, we get two sequences of representatives $\left(\widetilde{\psi}_{u}^{k}, \widetilde{\Gamma}_{u}\right)$ and $\left(\widetilde{\phi}_{k}, \widetilde{\Gamma}_{-u}\right)$ such that the volume growths exponentially faster in the first sequence than in the second one. The lemma follows since, by Lemma 9.7, the matrices associated to the representatives obtained after collapsing $B_{u}$ are irreducible (a non-negative, integer matrix $M$ is irreducible if for any $(i, j)$, there is $N_{i j}$ such that the $(i, j)$-coefficient of $M^{N_{i j}}$ is positive).

Proof of Proposition 9.1. Let $\mathcal{W}$ be a standard dynamical branched surface with a positive cocycle representing $\alpha \in \operatorname{Out}\left(\mathbb{F}_{n}\right)$. Up to passing to a positive power $\alpha^{k}$ of $\alpha$, Lemma 9.2 gives a standard dynamical branched surface $\mathcal{V}$ for which subsequent lemmas apply. Let $m \geq 1$ be the integer given by Lemma 9.8, and let $\left(\psi_{m}, \tau\right)$ and $\left(\phi_{m}, \tau\right)$ be the two representatives given there. They respectively represent $\alpha^{k m}$ and $\alpha^{-k m}$. Since $\left(\psi_{m}, \tau\right)$ is an invariant train-track, $\lambda\left(\psi_{m}\right)=\lambda\left(\alpha^{k m}\right)$, whereas $\lambda\left(\phi_{m}\right) \geq \lambda\left(\alpha^{-k m}\right)$. Lemma 9.8 then gives $\lambda\left(\alpha^{-k m}\right)<\lambda\left(\alpha^{k m}\right)$, with $k$ and $m$ positive integers. Thus $\lambda\left(\alpha^{-1}\right)<\lambda(\alpha)$ as announced.

\section{Proof of the theorems}

Proof of Theorem 3.3. $(b) \Rightarrow(a)$ is a consequence of

Proposition 10.1 ( $[\mathbf{1 6}, \mathbf{1 7}])$. Let $\alpha \in \operatorname{Out}\left(\mathbb{F}_{n}\right)$ be represented by a positive cocycle $u$ of a standard dynamical branched surface $\mathcal{W}$. Let us assume that $\mathcal{W}$ admits an embedding in some compact 3-manifold $M^{3}$. Let $M_{\mathcal{W}}$ be the regular neighborhood of $\mathcal{W}$ in $M^{3}$. Let $u$ be any positive cocycle of $\mathcal{W}$. Then: 
(a) $u$ defines a surface $S_{u}$, with fundamental group $\mathbb{F}_{n}$, which is properly embedded in $M_{\mathcal{W}}$;

(b) $S_{u}$ is the fiber of a fibration of $M_{\mathcal{W}}$ over $\mathbb{S}^{1}$;

(c) the monodromy of this fibration induces $\alpha$ on the fundamental group of $S_{u}$. Furthermore the monodromy is pseudo-Anosov and the associated foliations have no interior singularities.

The monodromy is pseudo-Anosov because no singular leaf connects $\partial M_{\mathcal{W}}$ to itself (see [17] for the construction of the contraction-dilation directions). This is indeed forbidden by the non-existence of annuli or Moebius-bands among the regions of $\mathcal{W}$ and by the compatibility of the orientation of the singular graph with the smoothing. All the other assertions are borrowed from $[16,17]$.

$(a) \Rightarrow(d)$ : By Proposition 9.1, W can be embedded in a compact 3-manifold. By Proposition 8.1, the same standard 2-complex, equipped with the reverse orientation on $\mathcal{S}(\mathcal{W})$, admits a compatible structure of dynamical branched surface $\mathcal{V}$. If $u$ is a positive cocycle of $\mathcal{W}$ representing $\alpha$, the cocycle $-u$ of $\mathcal{V}$ represents $\alpha^{-1}$.

$(d) \Rightarrow(c)$ : Assume that $\alpha$ is not geometric. Then $\mathcal{W}$ does not embed in a $3-$ manifold. Thus $\lambda\left(\alpha^{-1}\right)<\lambda(\alpha)$ by Proposition 9.1. If $\alpha$ is not geometric, neither is $\alpha^{-1}$ so that the same arguments applied to $\alpha^{-1}$ give $\lambda\left(\left(\alpha^{-1}\right)^{-1}\right)=\lambda(\alpha)<\lambda\left(\alpha^{-1}\right)$ whence a contradiction. It follows that $\alpha$ is geometric so that $\lambda(\alpha)=\lambda\left(\alpha^{-1}\right)$.

$(c) \Rightarrow(b)$ is given by Proposition 9.1 .

Proof of Theorem 1.1. Call nice any outer automorphism of $\mathbb{F}_{n}$ which is in

$$
\operatorname{NTT}\left(\mathbb{F}_{n}\right)=\operatorname{SDBS}\left(\mathbb{F}_{n}\right)
$$

The theorem is then implied by Theorem 3.3 and Proposition 10.1.

Proof of Theorem 5.12. The equivalences $(a) \Leftrightarrow(c) \Leftrightarrow(d)$ are a straightforward consequence of Theorems 3.3 and 7.1. The implication $(a) \Rightarrow(b)$ is a consequence of the combinatorial suspension explicited while proving Proposition 7.2 (see Remark 7.6), and of the equivalence between the items (a) and (b) of Theorem 3.3.

Let us prove $(b) \Rightarrow(a)$. Let $(\psi, \tau)$ be a nice train-track for $\alpha$. Let $\mathcal{B}$ be a set of loops in $\tau$ homotopically preserved by $\psi$, as given by item (b) (we call "set of $\partial$-loops" such a set of loops). The combinatorial suspension described for proving Proposition 7.2, applied to $(\psi, \tau)$, gives a standard dynamical branched surface $\mathcal{W}$ with a positive cocycle $u$ defining $(\psi, \tau)$ as the return-map $\psi$ of some nonsingular semi-flow on a cross-section $\tau$ in $\mathcal{W}$ (see Remark 7.6). Assume that $\alpha$ is not geometric. Then, by Theorem 3.3, $\mathcal{W}$ cannot be embedded in a 3-manifold. By Lemma 8.6, some region $R$ of $\mathcal{W}$ admits an odd number of mirrors in its boundary. Let $s$ and $a$ be the source and sink of $R$. Assume that $\tau$ is embedded in $\mathcal{W}$ with an edge $e$ connecting the two incoming edges at $s$. The loops in $\mathcal{B}$ pass over $e$ either like in the trivial I-bundle over $e$ (left of Figure 10.1) or like the twisted $I$-bundle over $e$ (right of Figure 10.1). 

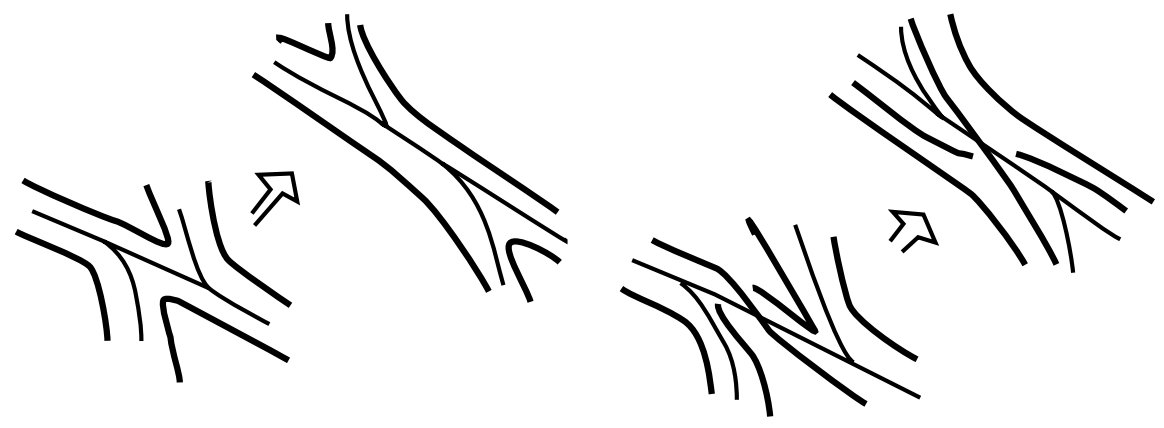

Figure 10.1. Preservation and Non-Preservation of $\partial$-loops.

Assume that this last case is satisfied. Then, as illustrated by Figure 10.1, the reduced image of $\mathcal{B}$ under the semi-flow is not a set of $\partial$-loops and pass four times over the edge of the new train-track which connects the two outgoing edges at $s$. Since the four subpaths are legal subpaths, any further image of $\mathcal{B}$ also crosses at least four times the edge intersecting the cycle of $\mathcal{W}$ through $s$. It follows that the reduced image of $\mathcal{B}$ under $\psi$ passes also at least four times over the edge $e$, thus $\mathcal{B}$ is not homotopically preserved by $\psi$, which is a contradiction with our assumption.

Assume now that the loops in $\mathcal{B}$ pass over $e$ like the trivial I-bundle. Then this is also true for their reduced images under the Whitehead-move through $s$ (see Figure 10.1). Pushing further along the semi-flow, since $R$ has an odd number of mirrors in its boundary, we get a train-track $\tau^{\prime}$ with an edge $f$ connecting the two incoming edges at $a$, and such that the reduced image of $\mathcal{B}$ in $\tau^{\prime}$ is a set of $\partial$-loops which pass over $f$ like the twisted $I$-bundle. This implies, as before, that $\mathcal{B}$ is not homotopically preserved when pushing through $a$.

It only remains to check that we can assume that some edge $e$ of $\tau$ connects the two incoming edges at $s$. If this is not satisfied by $\tau$, then it is satisfied by the invariant train-track $\left(\psi^{\prime}, \tau^{\prime}\right)$ obtained after pushing $\tau$ through some vertices of $\mathcal{W}$ - see Section 6. Moreover, the same arguments as those exposed above, about the preservation or non-preservation of a set of $\partial$-loops when pushing through a vertex of $\mathcal{W}$, give a set of $\partial$-loops in $\tau^{\prime}$ homotopically preserved under $\psi^{\prime}$. The conclusion follows as above, by substituting $\left(\psi^{\prime}, \tau^{\prime}\right)$ to $(\psi, \tau)$.

\section{A. Appendix: From train-tracks with circuits to nice train-tracks}

Let $\psi: \Gamma \rightarrow \Gamma$ be a tight graph-map, i.e. the image of any edge is a locally injective path, and let $(p, q)$ be a cancellation-pair for $\psi$.

A $B H$-folding [2] at $(p, q)$ from $(\psi, \Gamma)$ to $(\widehat{\psi}, \widehat{\Gamma})$ is defined by: $\widehat{\psi} \circ \pi=\pi \circ \psi$, $\widehat{\Gamma}$ is the graph obtained from $\Gamma$ by folding at $(p, q)$ and $\pi: \Gamma \rightarrow \widehat{\Gamma}$ is the associated quotient-map.

An unfolding [21] from a tight graph-map $(\widehat{\psi}, \widehat{\Gamma})$ to a tight graph-map $(\psi, \Gamma)$ is a homotopic right-inverse of a $\mathrm{BH}$-folding from $(\psi, \Gamma)$ to $(\widehat{\psi}, \widehat{\Gamma})$, i.e. for any 
edge $e$ of $\Gamma,(\widehat{\psi} \circ \pi)(e)=(\pi \circ \psi)(e)$ up to pulling-tight. An efficient unfolding is an unfolding from a invariant train-track to an invariant train-track.

Let $(\psi, \tau)$ be a train-track with circuits. Consider a cancellation-pair $\left(p_{i}, q_{i}\right)$ associated to a cancellation-path $w_{i}$. Its tip, i.e. the common vertex of $p_{i}$ and $q_{i}$, is the terminal vertex of a cancellation-path, noted $w_{i-1}$. Associated to $w_{i-1}$ is another cancellation-pair, denoted by $\left(p_{i-1}, q_{i-1}\right)$. Eventually, repeating the process, we go back to $\left(p_{i}, q_{i}\right)$. The cyclically ordered sequence of cancellation-pairs, or of the associated cancellation-paths, is called a circuit.

The circuits exhibited above allow us to efficiently unfold, as long as we wish, any train-track with circuits, starting from any tip of cancellation-pair.

Lemma A.1. Let $(\psi, \tau)$ be a train-track with circuits. If any two cancellationpairs in bad position belong to two distinct circuits then a finite sequence of efficient unfoldings transforms $(\psi, \tau)$ to a nice train-track.

Proof. Let us consider two cancellation pairs $\left(p_{i}, q_{i}\right),\left(p_{j}, q_{j}\right)$ in bad position. Without loss of generality $p_{i}=w p_{j} \cdots$ and $w$ does not contain the tip of a cancellation-pair $\left(p_{k}, q_{k}\right)$ with $p_{i}=\cdots p_{k} \cdots$. We unfold at the tip of $\left(p_{j}, q_{j}\right)$ along a legal path which is the concatenation of a proper subset of an edge with the path $w$. This suppresses the considered pair in bad position. This might create a new one if, by this unfolding, we put the cancellation-pair $\left(p_{j-1}, q_{j-1}\right)$ preceding $\left(p_{j}, q_{j}\right)$ in its circuit, in bad position with respect to some $\left(p_{k}, q_{k}\right)$. If this happens we iterate the process. Thanks to the assumption that no two cancellation-pairs in bad position lie in a same circuit, we eventually end with a train-track map with one couple less of cancellation-pairs in bad position. Lemma A.1 readily follows.

Corollary A.2. If $\alpha \in \operatorname{Out}\left(\mathbb{F}_{n}\right)$ is represented by a train-track with circuits, then there is an integer $1 \leq k \leq(2 n-2)$ ! such that $\alpha^{k}$ is represented by a nice traintrack.

Proof. Let $(\psi, \tau)$ be a train-track with circuits of $\alpha$. Setting $k$ equal to the $1 \mathrm{~cm}$ of the lengths of the circuits of $(\psi, \tau),\left(\psi^{k}, \tau\right)$ admits a collection of length 1 circuits for which it is a train-track with circuits. Lemma A.1 gives a nice train-track for $\left(\psi^{k}, \tau\right)$.

Thanks to Theorem 5.12, the above corollary gives the following statement:

Theorem A.3. Let $\alpha \in \operatorname{Out}\left(\mathbb{F}_{n}\right)$ admitting a train-track with circuits. Then the following properties are equivalent for some $1 \leq k \leq(2 n-2)$ ! :

(a) $\alpha^{k}$ is geometric.

(b) $\lambda(\alpha)=\lambda\left(\alpha^{-1}\right)$.

(c) both $\alpha^{k}$ and $\alpha^{-k}$ admit a nice train-track.

Remark A.4. As can be observed by looking at the proof of Corollary A.2, (2n$2)$ ! is only a rough upper-bound on $k$.

The best upper-bound is $\max _{n_{1}+\cdots+n_{i}=2 n-2} \operatorname{lcm}\left(n_{1}, \cdots, n_{i}\right)$. 


\section{B. Appendix: Finding train-tracks with circuits}

To be able to search for train-track with circuits representatives among the representatives of an outer free group automorphism, we need the "connectedness", in a sense to be defined, of the set of efficient representatives of an outer free group automorphisms. Here, an efficient representative of $\alpha \in \operatorname{Out}\left(\mathbb{F}_{n}\right)$ is a representative $(\psi, \Gamma)$ of $\alpha$ such that for any $k \geq 1, \psi^{k}$ is locally injective when restricted to the edges. It is easily checked that an invariant train-track of $\alpha$ is an efficient representative of $\alpha$. Usually, efficient representatives are required to map vertices to vertices, which is not the case here but this is a fairly unimportant point.

Once the connectedness established, it would not be hard to describe a finite algorithm. Unfortunately, the state of art about the connectedness of the set of efficient representatives is very unsatisfactory at the moment of the writing of this paper.

- In [21], the connectedness is asserted in the case of an irreducible automorphism and with respect to certain moves (essentially, BH-folding and efficient unfoldings). However there is a slight mistake in [21]. This mistake should be corrected by the introduction of global BH-foldings and global unfoldings: a global (un)-folding consists of (un)-folding simultaneously all the cancellationpairs of a same circuit. On the other hand, the assumption of irreducibility seems needed there only to ensure the existence of an efficient representative.

- If the automorphism is Irreducible With Irreducible Powers (IWIP), the connectedness under the above moves is a consequence of [22]. The methods of this last paper are completely different from the previous one.

- In [14], the connectedness is established for the set of efficient representatives of an outer free group automorphism induced by a pseudo-Anosov of a surface, which are embedded in the surface. The connectedness is established with respect to the same kind of moves as above, but which are also embedded in the surface. This does not mean that the whole set of efficient representatives is connected under these moves (even non-embedded ones): it might exist nonembedded efficient representatives which could not be joined to any embedded one.

\section{Appendix: Characterizing mapping-tori of pseudo-Anosov homeomorphisms}

Proposition C.1. Let $S$ be a compact surface with boundary. Let h be any pseudoAnosov homeomorphism of $S$, with no interior singularities. The outer free group automorphism $\alpha$ induced by $h$ on the fundamental group of $S$ is represented by a train-track with circuits. If, in addition, $S$ is orientable and $h$ is orientationpreserving, then $\alpha$ admits a nice train-track representative.

We refer the reader to [26] and [8] for all what is needed about pseudo-Anosov homeomorphisms. 
Proof. Any outer automorphism $\alpha$ induced by a pseudo-Anosov without interior singularities is represented by an invariant train-track $(\psi, \tau)$ which admits a set of cancellation-paths. This is a well-known and easy consequence of the existence of the singular leaves. These leaves give the way to unfold at the vertices of the train-track to get the cancellation-paths.

Now, consider an oriented edge $e$. Since any train-track of a pseudo-Anosov homeomorphism is recurrent (i.e. carries a measure with positive weights), there are two legal edge-paths $p_{+}, p_{-}$in $\tau$ beginning at the 2-sheeted side of their initial vertices and ending respectively with $e$ and $e^{-1}$. By the dilation property of a pseudoAnosov homeomorphism, the lengths of the legal paths composing a cancellationpair for $\left(\psi^{j}, \tau\right)$ tend toward infinity with $j$. Thus, there is $j \geq 1$ such that both $p_{+}$ and $p_{-}$belong to cancellation-pairs of $\left(\psi^{j}, \tau\right)$ so that $(\psi, \tau)$ is a train-track with circuits.

When the surface is orientable and the homeomorphism is orientation-preserving, two cancellation-pairs in bad position do not belong to a same circuit. Lemma A.1 gives the second assertion of the proposition.

Theorem C. 2 below is then a straightforward consequence of our process of combinatorial suspension:

Theorem C.2. Let $S$ be an orientable compact surface with boundary. Let $h$ be an orientation-preserving pseudo-Anosov homeomorphism of $S$, with no interior singularities. Then the mapping torus of $(h, S)$ admits as a spine a standard dynamical branched surface $\mathcal{W}$ carrying the weak unstable foliation of the suspension flow. Moreover $\mathcal{W}$ admits a positive cocycle $u \in C^{1}(W ; \mathbb{Z})$ associated to the fibration over the circle with fiber $S$ and monodromy the isotopy-class of $h$. This cocycle defines an invariant train-track for $h$.

The weak unstable foliation is the foliation tangent at each point to the plane, in the tangent space of the manifold, spanned by the neutral and unstable directions of the flow.

\section{Appendix: Examples}

\section{D.1. Example 0: Two automorphisms which do not admit train-tracks with circuits representatives}

We borrow the two examples from [20] (the two are IWIP). The first one, denoted by $\beta$ and represented by $(g, G)$, is defined as follows: the graph $G$ has two vertices $r, q$, four edges $B, C, D, E$ with $B$ from $q$ to $r, C$ and $D$ from $r$ to $q, E$ from $q$ to $q$; the map $g$ is defined by $g(B)=C E^{-1} C^{-1} D E, g(C)=C^{-1} B^{-1} E^{-1} D^{-1}$, $g(D)=B, g(E)=C B$. The three turns $(C, B),\left(C, E^{-1}\right)$ and $(E, B)$ at the vertex $q$ are crossed respectively by $g(E), g(B)$ and $g(C)$. This forbids to unfold $(g, G)$ by keeping the efficiency of the map. Thus $\beta$ does not admit a train-track with 
circuits (thanks to the connectedness evoked in Appendix B which holds here since $\beta$ is an IWIP).

The second example is $\gamma \in \operatorname{Aut}\left(\mathbb{F}_{3}\right)$ given by $\gamma(A)=B, \gamma(B)=C$ and $\gamma(C)=B^{-1} A$, with $\mathbb{F}_{3}=<A, B, C>$. This is the automorphism of lowest growth-rate in the pair $\left\{\gamma, \gamma^{-1}\right\}$. We consider the representative on the rose with three petals, $A, B$ and $C$. Consider the illegal turn $\left(A^{-1}, C\right)$. The turns $\left(B, A^{-1}\right)$, $\left(C^{-1}, B\right),\left(C^{-1}, B^{-1}\right)$ and $\left(A, C^{-1}\right)$ are crossed by $\gamma^{7}(B), \gamma^{3}(B), \gamma^{8}(B)$ and $\gamma^{6}(B)$. This forbids to unfold $\left(A^{-1}, C\right)$ in whatever direction by still keeping an efficient map. Therefore, for the same reason as $\beta, \gamma$ cannot be represented a traintrack with circuits.

Figure D.1 presents the elementary-map used to compute the invariant traintracks in the examples below. It makes the computations easier than the elementarymap given for proving Proposition 9.1.

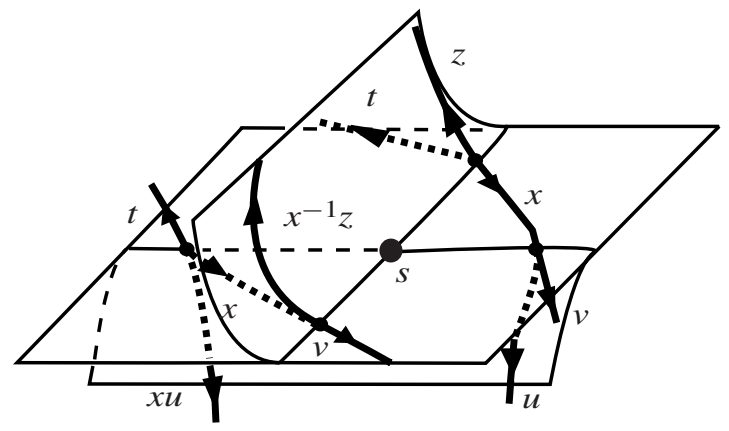

Figure D.1. Elementary-map.

\section{D.2. Example 1: Figure D.2}

We consider the standard dynamical branched surface illustrated by Figure D.2. The positive cocycle $u(0)=1, u(1)=3$ defines the invariant train-track $\left(\psi_{1}, \tau_{1}\right)$ where $\tau_{1}$ is drawn in this figure, and $\psi_{1}$ is given by : $\psi_{1}(A)=C, \psi_{1}(C)=A E$, $\psi_{1}(E)=H, \psi_{1}(H)=I, \psi_{1}(I)=A^{-1} K, \psi_{1}(K)=A$. The automorphism of $\mathbb{F}_{3}=<X, Y, Z>$ it represents is $\alpha_{1}(X)=Y, \alpha_{1}(Y)=Z^{-1} X, \alpha_{1}(Z)=X$. There is one mirror on the edge 0 so that there is an odd number of mirrors in the boundary of the unique region of $\mathcal{W}$. Therefore $\mathcal{W}$ cannot be embedded in a 3-manifold and thus $\alpha_{1}$ is not geometric. This is checked directly by computing the growth-rate: $\lambda\left(\alpha_{1}\right) \simeq 1,32$ whereas $\lambda\left(\alpha_{1}^{-1}\right) \simeq 1,16$ (up to $10^{-2}$ ).

Some words about this example: We can slightly modify this example by putting mirrors on both edges of the singular graph. We get a unique region with boundary " $0,0,1,0^{-1}, 1^{-1}, 1^{-1}$ ". This is also a standard dynamical branched surface, and it admits as positive cocycle $u(0)=u(1)=1$. This cocycle defines an automorphism of $\mathbb{F}_{2}$. These automorphisms are known to be geometric and, indeed, the 
branched surface embeds in a non-orientable 3-manifold. The author did not think immediately to these so simple (too simple ...) examples, but became aware of them thanks to John Crisp, who studied these 2-complexes with other perspectives in mind [6].

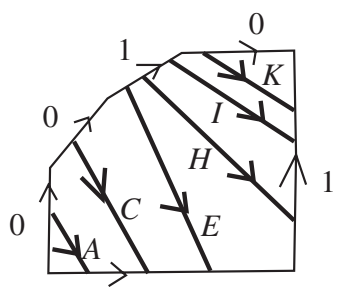

1
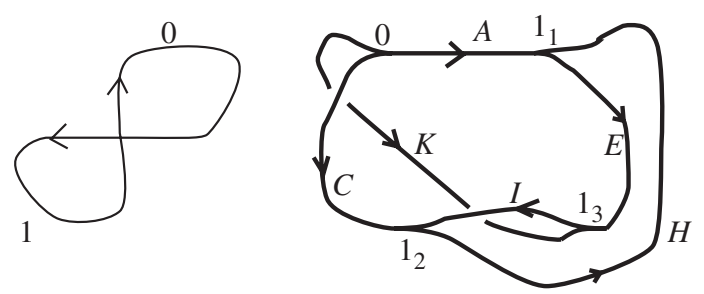

Figure D.2. A "simple" non geometric automorphism.

\section{D.3. Example 2: Figure D.3}

We consider the invariant train-track $\left(\psi_{2}, \tau_{2}\right)$ with $\tau_{2}$ being represented in the righthand side of Figure D.3 and $\psi_{2}$ being defined by: $\psi_{2}(A)=E A C, \psi_{2}(C)=$ $I E K A^{-1}, \psi_{2}(E)=H, \psi_{2}(H)=A K^{-1} E^{-1} I^{-1}, \psi_{2}(I)=E^{-1} I^{-1}, \psi_{2}(K)=$ $E A K^{-1} E^{-1} I^{-1}$.

It represents $\alpha_{2} \in \operatorname{Aut}\left(\mathbb{F}_{3}\right), \mathbb{F}_{3}=<X, Y, Z>$, defined by $\alpha_{2}(X)=X$, $\alpha_{2}(Y)=Y Z Y^{-1} X$ and $\alpha_{2}(Z)=Z^{-1} Y^{-1}$.

A first way to check that $\alpha_{2}$ is geometric: try all the sets of reduced loops in $\tau_{2}$ whose union crosses exactly twice each edge. There are only a finite number of them. Compute whether they are homotopically preserved or not by $\psi_{2}$. There is such a set preserved, this is the set of boundary loops of the trivial thickening of $\tau_{2}$ embedded in the plane as illustrated on the right-hand side of Figure D.3, i.e. the surface is the three times punctured disc.

A second way: the combinatorial suspension yields the standard dynamical branched surface $\mathcal{W}_{2}$ with positive cocycle and r-embedded graph illustrated by Figure D.3. Check whether $\mathcal{W}_{2}$ admits an embedding in a compact 3-manifold (see the criteria given in $8.6,8.1$, or 8.7 ).

To obtain the surface where to realize $\alpha_{2}$, just read-off, once local embeddings of $\mathcal{W}_{2}$ in $\mathbb{R}^{3}$ have been chosen, the cyclic orderings that they define at the vertices of the r-embedded graph, and whether over each edge the surface is the trivial or twisted I-bundle. All is deduced from the combinatorics of the complex.

Some words about this example: the automorphism is in fact in the same class than the braid automorphism $\sigma_{1} \sigma_{2}^{-1}$ of $\mathcal{B}_{3}$. The example was constructed from the "usual" efficient representative $(g, G)$ where $G$ consists of four 3-valent vertices and six edges: three, $d, e, f$, are loops and the three others, $a, b, c$, connect the vertices of $d, e, f$ to the fourth vertex. The map $g$ is defined by $g(a)=a d a^{-1} c$, $g(b)=a, g(c)=c f^{-1} c^{-1} b, g(d)=f, g(e)=d$ and $g(f)=e$. To construct $\mathcal{W}_{2}$, 
we unfolded until obtaining $\left(\psi_{2}, \tau_{2}\right)$. Observe that the invariant train-track defined by the given positive cocycle is not $\left(\psi_{2}, \tau_{2}\right)$ but $\left(\psi_{2}^{\prime}, \tau_{2}\right)$ with $\psi_{2}^{\prime}(A)=A C I$, $\psi_{2}^{\prime}(C)=E K, \psi_{2}^{\prime}(E)=H E, \psi_{2}^{\prime}(H)=K^{-1} E^{-1} I^{-1}, \psi_{2}^{\prime}(I)=A^{-1} E^{-1} I^{-1}$ and $\psi_{2}^{\prime}(K)=A K^{-1} E^{-1}$.

There are many other positive cocycles, and so many other free group automorphisms, represented by this branched surface: for instance $u(6)=u(7)=u(1)=$ $1, u(3)=2, u(4)=3$ and $u(x)=0$ for the other edges. All are geometric.
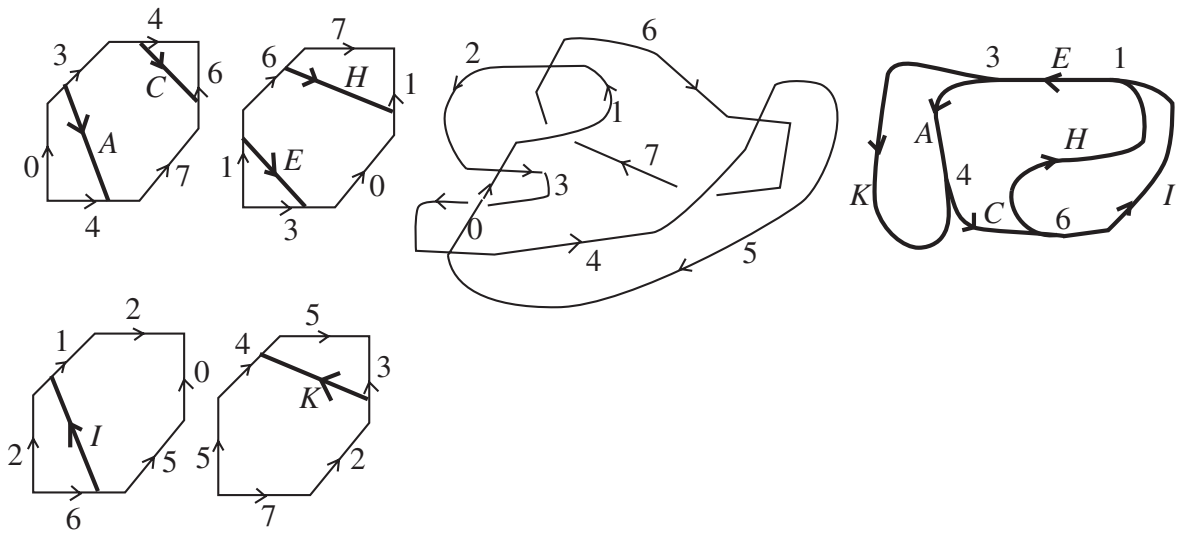

Figure D.3. A geometric reducible automorphism.

\section{D.4. Example 3: Figure D.4}

Let $\alpha_{3} \in \operatorname{Aut}\left(\mathbb{F}_{5}\right), \mathbb{F}_{5}=<X, Y, Z, T, U>$, defined by: $\alpha_{3}(X)=Y, \alpha_{3}(Y)=Z$, $\alpha_{3}(Z)=T, \alpha_{3}(T)=U, \alpha_{3}(U)=T X^{-1}$.

This is the automorphism represented by the standard branched surface $\mathcal{W}_{3}$ and positive cocycle of Figure D.4. It admits as invariant train-track $\left(\psi_{3}, \tau_{3}\right), \tau_{3}$ being in the right-hand side of the figure, and $\psi_{3}$ is defined by: $\psi_{3}(A)=B, \psi_{3}(B)=C A$, $\psi_{3}(C)=D, \psi_{3}(D)=E, \psi_{3}(E)=F, \psi_{3}(F)=G, \psi_{3}(G)=H K^{-1} A^{-1}$, $\psi_{3}(H)=I K, \psi_{3}(I)=J, \psi_{3}(J)=A, \psi_{3}(K)=L, \psi_{3}(L)=K$.

The edge 1 of $\mathcal{S}\left(\mathcal{W}_{3}\right)$ does not appear in one-to-last position whatever maximal positive path in the boundary of a region is considered. Thus $\mathcal{W}_{3}$ cannot be embedded in a compact 3-manifold so that $\alpha_{3}$ is not geometric.

Some words about this example: computing $\alpha_{3}^{-1}$ is very easy. One has $\alpha_{3}^{-1}(X)=$ $Z U, \alpha_{3}^{-1}(Y)=X, \alpha_{3}^{-1}(Z)=Y, \alpha_{3}^{-1}(T)=Z, \alpha_{3}^{-1}(U)=T$. This is a positive automorphism so that the map on the rose with petals $\{X, Y, Z, T, U\}$ is an efficient representative. One thus obtains $\lambda\left(\alpha_{3}^{-1}\right) \simeq 1.19$, whereas $\lambda\left(\alpha_{3}\right) \simeq 1.23$ (up to $\left.10^{-2}\right)$. 

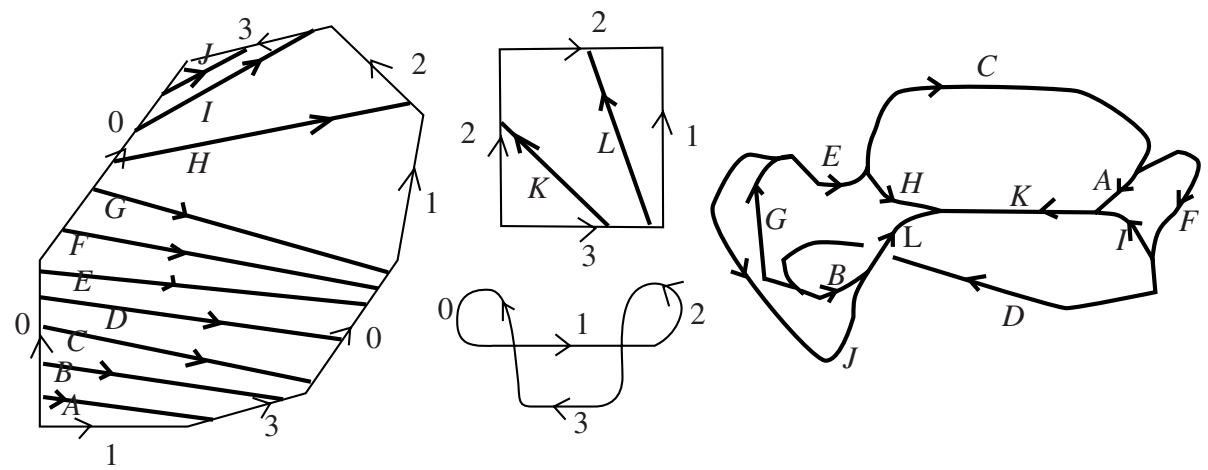

Figure D.4. A non-geometric automorphism.

\section{D.5. Example 4: Figure D.5}

We consider the automorphism $\alpha_{4} \in \operatorname{Aut}\left(\mathbb{F}_{4}\right)$ given by $\alpha_{4}(X)=Y X^{-1} Z^{-1} Y^{-1}$, $\alpha_{4}(Y)=X Z^{-1} Y^{-1}, \alpha_{4}(Z)=Y Z X^{-1} T Y^{-1}$ and $\alpha_{4}(T)=X^{2} Z^{-1} Y^{-1}$, with $\mathbb{F}_{4}=<X, Y, Z, T>$. By unfolding, we find the nice train-track $\left(\psi_{4}, \tau_{4}\right)$ where $\tau_{4}$ is the train-track on the right-hand side of Figure D.5 and $\psi_{4}$ is defined by: $\psi_{4}(A)=C, \psi_{4}(C)=M I^{-1}, \psi_{4}(E)=H, \psi_{4}(H)=E A, \psi_{4}(I)=K M$, $\psi_{4}(K)=A^{-1} E^{-1} L, \psi_{4}(M)=N, \psi_{4}(N)=A M, \psi_{4}(L)=E A M I^{-1} A M I^{-1}$. Our combinatorial suspension yields the standard dynamical branched surface of Figure D.5 with the positive cocycle $u(0)=u(2)=2, u(4)=u(7)=1$ and $u(x)=0$ for the other edges $x$ of the singular graph. This branched surface can be embedded in a compact 3-manifold so that $\alpha_{4}$ is geometric. The surface where to realize $\alpha_{4}$ is the trivial thickening of $\tau_{4}$ which has been drawn with the correct cyclic ordering at the vertices. This is the orientable genus 2 surface with one boundary component.
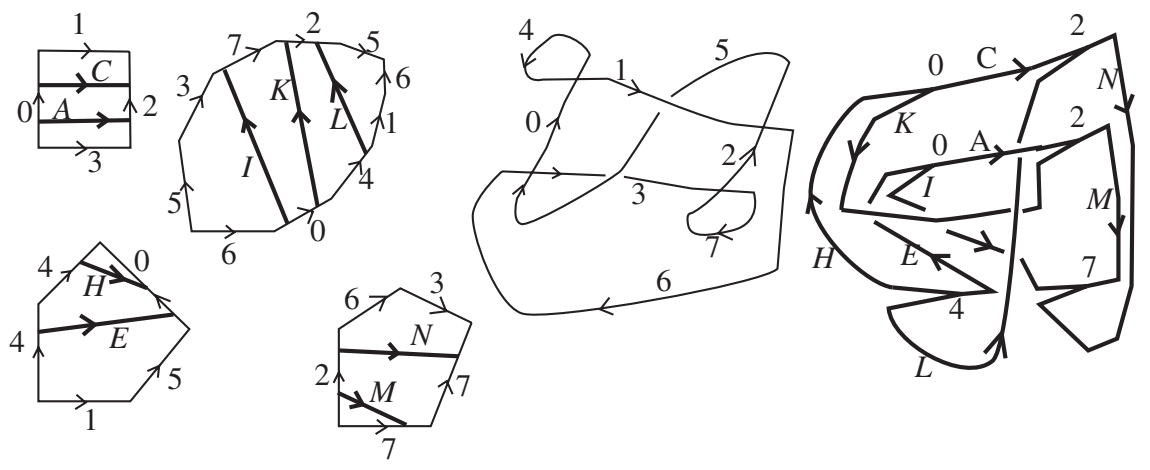

Figure D.5. An IWIP geometric automorphism. 


\section{References}

[1] M. Bestvina, M. Feighn and M. Handel, The Tits alternative for $\operatorname{Out}\left(() F_{n}\right)$ : $D y$ namics of exponentially growing free group automorphisms, Ann. of Math. 151 (2000), $517-623$.

[2] M. Bestvina and M. HAndel, Train-tracks and free group-automorphisms, Ann. of Math. 135 (1992), 1-52.

[3] M. Bestvina and M. HAndel, Train-tracks for surface homeomorphisms, Topology 34 (1995), 109-140.

[4] R. Benedetti and C. Petronio, A finite graphic calculus for 3-manifolds, Manuscripta Math. 88 (1995), 291-310.

[5] R. Benedetti and C. Petronio, "Branched Standard Spines of 3-Manifolds", Lectures Notes in Mathematics, 1653, Springer, Berlin, 1997.

[6] N. BRADY and J. CRISP, CAT(0) and CAT(-1) dimensions of torsion free hyperbolic groups, to appear in Comment. Math. Helv.

[7] B. G. CASLER, An imbedding theorem for connected 3-manifolds with boundary, Proc. Amer. Math. Soc. 16 (1965), 559-566.

[8] A. J. CASSON and S. A. Bleiler "Automorphisms of Surfaces After Nielsen and Thurston", London Mathematical Society Student Texts 9, Cambridge University Press, Cambridge, 1988.

[9] J. CHRISTY, Branched surfaces and attractors I: Dynamic branched surfaces, Trans. Amer. Math. Soc. 336 (1993), 759-784.

[10] H. D. Coldewey, E. Vogt and H. Zieschang, "Surfaces and Planar Discontinuous Groups", Lecture Notes in Mathematics 835, Springer-Verlag, 1980.

[11] D. J. COLLINS and H. ZIESCHANG, Combinatorial group theory and fundamental groups, In: “Algebra VII”, Encyclopaedia Math. Sci., Vol. 58, Springer, Berlin, 1993, 1-166.

[12] W. DiCKS and E. VentURA, Irreducible automorphisms of growth rate one, J. Pure Appl. Algebra 88 (1993), 51-62.

[13] A. Fathi, F. Laudenbach and V. Poenaru, "Travaux de Thurston sur les Surfaces", Astérique 66-67, 1979.

[14] J. Fehrenbach, "Quelques Aspects Géométriques et Dynamiques du Mapping-Class Group", PhD dissertation, Université de Nice, Sophia Antipolis, 1998.

[15] D. Gaboriau, A. Jaeger, G. LeVitT and M. Lustig, An index for counting fixed points of automorphisms of free groups, Duke Math. J. 93 (1998), 425-452.

[16] F. Gautero, Dynamical 2-complexes, Geom. Dedicata 88 (2001), 283-319.

[17] F. Gautero, Cross-sections to semi-flows on 2-complexes, Ergod. Theory Dyn. Syst. 23 (2003), 143-174.

[18] S. GERSTEN, Geometric automorphisms of a free group of rank at least three are rare, Proc. Amer. Math. Soc. 89 (1983), 27-31.

[19] V. GuIRARDEL, Core and intersection number for group action on trees, Ann. Sci. Ecole Norm. Sup. 38 (2005), 847-888.

[20] M. HANDEL and L. Mosher, Parageometric outer automorphisms of free groups, Trans. Amer. Math. Soc. 359 (2007), 3153-3183.

[21] J. Los, On the conjugacy problem for automorphisms of free groups, With an addendum by the author, Topology 35 (1996), 779-808.

[22] J. LOS and M. LUSTIG, The set of train-track representatives of an irreducible free group automorphisms is contractible, http://www.crm.es/Publications/Preprints04.htm (2004).

[23] J. LOS and Z. NITECKI, Edge-transitive graph automorphisms and periodic surface homeomorphisms, Internat. J. Bifur. Chaos Appl. Sci. Engrg. 9 (1999), 1803-1813.

[24] M. LUSTIG, Structure and conjugacy for automorphisms of free groups I, II, Max-PlanckInstitut für Mathematik, Preprint Series 130 (2000) and 4 (2001).

[25] S. V. MatveEV, Special spines of piecewise linear manifolds, Math. USSR Sb. 21 (1973). 
[26] R. Penner and J. Harer, "Combinatorics of Train-Tracks", Annals of Mathematical Studies 125, Princeton University Press, 1991.

[27] J. R. Stallings, Topologically unrealizable automorphisms of free groups, Proc. Amer. Math. Soc. 84 (1982), 21-24.

[28] J. R. STALlings, Topology of finite graphs, Invent. Math. 71 (1983), 551-565.

[29] R. F. Williams, Expanding attractors, Inst. Hautes Étud. Sci. Publ. Math. 43 (1974), 169-203.

Université Blaise Pascal

Campus des Cézeaux

Laboratoire de Mathématiques

63177 Aubière, France

Francois.Gautero@math.univ-bpclermont.fr 